BULLETIN (New Series) OF THE

AMERICAN MATHEMATICAL SOCIETY

Volume 47, Number 4, October 2010, Pages 639-670

S 0273-0979(2010)01306-9

Article electronically published on August 3, 2010

\title{
ON ADDING A LIST OF NUMBERS (AND OTHER ONE-DEPENDENT DETERMINANTAL PROCESSES)
}

\author{
ALEXEI BORODIN, PERSI DIACONIS, AND JASON FULMAN
}

\begin{abstract}
Adding a column of numbers produces "carries" along the way. We show that random digits produce a pattern of carries with a neat probabilistic description: the carries form a one-dependent determinantal point process. This makes it easy to answer natural questions: How many carries are typical? Where are they located? We show that many further examples, from combinatorics, algebra and group theory, have essentially the same neat formulae, and that any one-dependent point process on the integers is determinantal. The examples give a gentle introduction to the emerging fields of one-dependent and determinantal point processes.
\end{abstract}

\section{INTRODUCTION}

Consider the task of adding a single column of digits:

\begin{tabular}{rrr}
7 & $\cdot$ & 7 \\
9 & $\cdot$ & 6 \\
4 & & 0 \\
8 & $\cdot$ & 8 \\
3 & & 1 \\
6 & & 7 \\
1 & $\cdot$ & 8 \\
6 & $\cdot$ & 4 \\
8 & & 2 \\
\hline 52 & &
\end{tabular}

We have put a dot to the right of a digit whenever the succeeding addition leads to a carry. The remainder $(\bmod 10)$ is written to the right of the dot. Thus $7+9=16$ results in a dot by the 7 and a remainder of 6 . At the end, the total is found by adding the number of dots (here, five) and appending the final remainder (here, 2) for a total of 52. The dots are a standard bookkeeping device used (for example) in the Trachtenberg system of speed addition [17.

How many carries are typical and how are they distributed? Common sense suggests that about half the places have carries and that if a carry occurs, it is less likely that the next addition gives a carry. Investigating these questions when digits are chosen uniformly at random leads to interesting mathematics.

Received by the editors May 1, 2009.

2010 Mathematics Subject Classification. Primary 05E15, 60B15.

(C)2010 American Mathematical Society Reverts to public domain 28 years from publication 
As additional motivation, look at the remainder column in the example above. Observe that there is a carry on the left (and so a dot) if and only if there is a descent on the right (a sequence $x_{1}, x_{2}, \ldots$ has a descent at $i$ if $x_{i}>x_{i+1}$ ). Further, if the original column of digits is independent and uniformly distributed, so is the remainder column. Thus, the results about carries give the distribution of the descent pattern in a random sequence of digits.

Section 2 derives the distribution theory of carries by elementary arguments. There are nice formulae for the chance of any pattern of carries, and the process is one-dependent, so a variety of probabilistic limit theorems is available.

Section 3 introduces determinantal point processes, shows that the carries process is determinantal, and illustrates how standard tools for determinantal point processes apply to carries.

Section 4 reviews the literature on stationary one-dependent processes, shows that all of these are determinantal and further that essentially all the neat formulae for carries and descents have versions for any stationary one-dependent process. Connections with symmetric function theory are developed. A large class of examples arising from the work of Polishchuk and Positselski 61] on Koszul algebras is shown to yield many natural examples (including the original carries process).

Section 5 gives combinatorial examples: descents in permutations from both uniform and non-uniform distributions, the connectivity set of a permutation, and binomial posets.

Section 6 generalizes from adding numbers to multiplying random elements in a finite group. For central extensions (e.g., the quaternions), there is again a carries process that is stationary, one-dependent, and determinantal. Thus explicit formulae and limit theorems are available.

Section 7 contains proofs of some of our more technical results. It shows that any one-dependent (possibly nonstationary) point process on the integers is determinantal. It also constructs a family of one-dependent determinantal processes (generalizing many examples in earlier sections), and computes its correlation kernel.

\section{Probability theory For CARRIES AND DESCEnts}

Throughout this section we work in base $b$ and so with the alphabet $\mathcal{B}=$ $\{0,1, \ldots, b-1\}$. Let $B_{1}, B_{2}, \ldots, B_{n}$ be a sequence of randomly chosen elements of $\mathcal{B}$ (independent and identically distributed). There is a descent at $i$ if $B_{i}>$ $B_{i+1}, 1 \leq i \leq n-1$. Let $X_{i}$ be 1 or 0 depending on whether or not there is a descent at $i$, and let $D=\left\{i: X_{i}=1\right\}$ be the descent set of $B_{1}, B_{2}, \ldots, B_{n}$.

The following probabilistic facts are elementary. They are stated for carries but verification is easier using descents.

Fact 1 (Single carries). For any $i \in[n-1]$,

$$
P\left(X_{i}=1\right)=\frac{1}{2}-\frac{1}{2 b}=\frac{\left(\begin{array}{l}
b \\
2
\end{array}\right)}{b^{2}}
$$

Thus, when $b=10$, the chance of a carry is .45 . When $b=2$, the chance of a carry is .25. For any base, $\operatorname{Var}\left(X_{i}\right)=\frac{1}{4}-\frac{1}{4 b^{2}}$. 
Fact 2 (Runs of carries). For any $i$ and $j$ with $1 \leq i<i+j \leq n$,

$$
P\left(X_{i}=X_{i+1}=\cdots=X_{i+j-1}=1\right)=\left(\begin{array}{c}
b \\
j+1
\end{array}\right) / b^{j+1} .
$$

Thus, a run of $b$ or more carries is impossible. Further,

$$
\operatorname{Cov}\left(X_{i}, X_{i+1}\right)=E\left(X_{i} X_{i+1}\right)-E\left(X_{i}\right) E\left(X_{i+1}\right)=-\frac{1}{12}\left(1-\frac{1}{b^{2}}\right) .
$$

Fact 3 (Stationary one-dependence). The distribution of $\left\{X_{i}\right\}_{i \in[n-1]}$ is stationary: for $J \subseteq[n-1], i \in[n-1]$ with $J+i \subseteq[n-1]$, the distribution of $\left\{X_{j}\right\}_{j \in J}$ is the same as the distribution of $\left\{X_{j}\right\}_{j \in J+i}$. Further, the distribution of $\left\{X_{i}\right\}_{i \in[n-1]}$ is one-dependent: if $J \subseteq[n-1]$ has $j_{1}, j_{2} \in J \Rightarrow\left|j_{i}-j_{2}\right|>1$, then $\left\{X_{j}\right\}_{j \in J}$ are jointly independent binary random variables with $P\left(X_{j}=1\right)=\frac{1}{2}-\frac{1}{2 b}$. The literature on $m$-dependent random variables is extensive. In particular, a classical central limit theorem 42 shows the following:

Theorem 2.1. For $n \geq 2$, the total number of carries $T_{n-1}=X_{1}+\cdots+X_{n-1}$ has mean $(n-1)\left(\frac{1}{2}-\frac{1}{2 b}\right)$, variance $\frac{n+1}{12}\left(1-\frac{1}{b^{2}}\right)$ and, normalized by its mean and variance, $T_{n-1}$ has a standard normal limiting distribution for $n$ large.

Remarks. (1) An $O\left(n^{-1 / 2}\right)$ error bound in this central limit theorem can be proved using the dependency graph approach to normal approximation by Stein's method [13], 19.

(2) Stationary pairwise independent processes can fail to obey the central limit theorem [8], 46].

Fact 4 ( $k$-point correlations). For $A \subseteq[n-1]$, let

$$
\rho(A)=P\left\{X_{i}=1 \text { for } i \in A\right\} .
$$

For $|A|=k$, the $\rho(A)$ are called $k$-point correlations for the point process $X_{1}, \ldots$, $X_{n-1}$. They are basic descriptive units for general point processes [18]. For the carries and descent process, they are simple to describe. Break $A \subseteq[n-1]$ into disjoint, nonempty blocks of consecutive integers $A=A_{1} \cup A_{2} \cup \cdots \cup A_{k}$. Thus $A=\{2,3,5,6,7,11\}=\{2,3\} \cup\{5,6,7\} \cup\{11\}$. From Facts 2 and 3 above,

$$
\rho(A)=\prod_{i=1}^{k}\left[\left(\begin{array}{c}
b \\
a_{i}+1
\end{array}\right) / b^{a_{i}+1}\right] \quad \text { if } A=\bigcup_{i=1}^{k} A_{i} \text { with }\left|A_{i}\right|=a_{i} .
$$

Fact 5 (Determinant formula). Let $\epsilon_{1}, \epsilon_{2}, \ldots, \epsilon_{n-1}$ be a fixed sequence in $\{0,1\}$. Then

$$
P\left\{X_{1}=\epsilon_{1}, \ldots, X_{n-1}=\epsilon_{n-1}\right\}=\frac{1}{b^{n}} \cdot \operatorname{det}\left(\begin{array}{c}
s_{j+1}-s_{i}+b-1 \\
b-1
\end{array}\right) .
$$

Here, if there are exactly $k 1$ 's in the $\epsilon$-sequence at positions $s_{1}<s_{2}<\cdots<s_{k}$, the determinant is of a $(k+1) \times(k+1)$ matrix with $(i, j)$ entry $\left(\begin{array}{c}s_{j+1}-s_{i}+b-1 \\ b-1\end{array}\right)$ for $0 \leq i, j \leq k$ with $s_{0}=0, s_{k+1}=n$.

Example 1. If $n=8, \epsilon_{1}=1, \epsilon_{2}=\epsilon_{3}=\epsilon_{4}=0, \epsilon_{5}=1, \epsilon_{6}=\epsilon_{7}=0$, the matrix is

$$
\left(\begin{array}{ccc}
\left(\begin{array}{c}
1+b-1 \\
b-1
\end{array}\right) & \left(\begin{array}{c}
5+b-1 \\
b-1
\end{array}\right) & \left(\begin{array}{c}
8+b-1 \\
b-1
\end{array}\right) \\
1 & \left(\begin{array}{c}
4+b-1 \\
b-1
\end{array}\right) & \left(\begin{array}{c}
7+b-1 \\
b-1
\end{array}\right) \\
0 & 1 & \left(\begin{array}{c}
3+b-1 \\
b-1
\end{array}\right)
\end{array}\right)
$$


When $b=2$, this is $\left(\begin{array}{lll}2 & 6 & 9 \\ 1 & 5 & 8 \\ 0 & 1 & 4\end{array}\right)$ with determinant 9 . Thus the chance of a carry at exactly positions 1 and 5 when adding eight binary numbers is $9 / 2^{8} \doteq .03516$. When $b=10$, this chance becomes $1,042,470 / 10^{8} \doteq .0104$. This is not so much smaller than in the binary case.

Proof of Fact 5. We follow Stanley [70, p. 69]. Let $\alpha_{n}(S)$ be the number of sequences with descent set contained in $S$ and $\beta_{n}(S)$ the number of sequences with descent set equal to $S$. Then

$$
\alpha_{n}(S)=\sum_{T \subseteq S} \beta_{n}(T), \quad \beta_{n}(S)=\sum_{T \subseteq S}(-1)^{|S-T|} \alpha_{n}(T) .
$$

There is a simple formula for $\alpha_{n}(S)$ : the number of weakly increasing sequences with entries in $\{0,1, \ldots, b-1\}$ of length $l$ is $\left(\begin{array}{c}l+b-1 \\ b-1\end{array}\right)$ using the usual stars and bars argument (place $b-1$ bars into $l+b-1$ places, put symbol 0 to the left of the first bar, symbol 1 between the first and second bar, and so on, with symbol $b-1$ to the right of the last bar). Then

$$
\alpha_{n}(S)=\left(\begin{array}{c}
s_{1}+b-1 \\
b-1
\end{array}\right)\left(\begin{array}{c}
s_{2}-s_{1}+b-1 \\
b-1
\end{array}\right) \cdots\left(\begin{array}{c}
n-s_{k}+b-1 \\
b-1
\end{array}\right),
$$

because any such sequence of length $n$ is constructed by concatenating nondecreasing sequences of length $s_{1}, s_{2}-s_{1}, \ldots, n-s_{k}$. Therefore,

$$
\beta_{n}(S)=\sum_{1 \leq i_{1}<i_{2}<\cdots<i_{j} \leq k}(-1)^{k-j} f\left(0, i_{1}\right) f\left(i_{1}, i_{2}\right) \cdots f\left(i_{j}, k+1\right)
$$

with $f(i, j)=\left(\begin{array}{c}s_{j}-s_{i}+b-1 \\ b-1\end{array}\right)$ (with $\left.s_{0}=0, s_{k+1}=n\right)$. From Stanley's discussion, $\beta_{n}(S)$ is the determinant of the $(k+1) \times(k+1)$ matrix with $(i, j)$ entry $f(i, j+1), 0 \leq$ $i, j \leq k$.

Example 2. From the above development, the chance that the sum of $n$ base $b$ digits has no carries is $\left(\begin{array}{c}n+b-1 \\ b-1\end{array}\right) / b^{n}$. When $n=8, b=2$, this is $9 / 2^{8} \doteq .03516$. When $n=8, b=10$, this is $\left(\begin{array}{c}17 \\ 9\end{array}\right) / 10^{8} \doteq .00024$. We feel lucky when adding eight numbers with no carries. These calculations show that we are lucky indeed.

\section{Determinantal Point PRocesses}

Let $\mathcal{X}$ be a finite set. A point process on $\mathcal{X}$ is a probability measure $P$ on the $2^{|\mathcal{X}|}$ subsets of $\mathcal{X}$. For example, if $\mathcal{X}=\{1,2, \ldots, n-1\}$, a point process is specified by recording where the carries occur in adding $n$ base $b$ numbers as in Section 2 . One simple way to specify $P$ is via its so-called correlation functions. For $A \subseteq \mathcal{X}$, let

$$
\rho(A)=P\{S: S \supseteq A\} .
$$

The collection of numbers $\{\rho(A)\}$ uniquely determine $P$ using inclusion/exclusion. A point process is determinantal with kernel $K(x, y)$ if

$$
\rho(A)=\operatorname{det}(K(x, y))_{x, y \in A} .
$$

On the right is the determinant of the $|A| \times|A|$ matrix with $(x, y)$ entry $K(x, y)$ for $x, y \in A$.

Determinantal point processes were introduced by Macchi [53 to model the distribution of fermions. See [18] for a textbook account, 68] for a survey, [43] for probabilistic developments and [5], [51] for many combinatorial examples. We 
warn the reader that these references mostly deal with symmetric kernels $K(x, y)$ whereas all of our examples involve nonsymmetric kernels.

There has been an explosive development of determinantal point processes over the past few years. Some reasons for this include:

(a) A raft of natural processes (including all those in the present paper) turn out to be determinantal. These include: the eigenvalues of various ensembles of random matrices (Dyson [26, 27, 28], Mehta [58]); the presence or absence of edges if a random spanning tree is chosen in a graph (Burton-Pemantle [11, Lyons [51]); random tilings and growth models (Johansson [47]); the structure of random partitions chosen from a variety of measures (e.g., the Plancherel measure on the symmetric group $S_{n}$ ) (Borodin-OkounkovOlshanski [6]); random dimers on bipartite planar graphs (Kenyon [49]); and the zeros of a random analytic function $\sum_{n=0}^{\infty} a_{n} z^{n}$ with i.i.d. complex Gaussian coefficients $a_{n}$ [59. The list goes on extensively.

(b) Specifying a kernel may be much easier than specifying a measure on all $2^{\mid \mathcal{X X}}$ subsets. Further, the correlation functions allow an easy computation of quantities of interest. For example, if $X_{x}$ is 1 or 0 depending on whether the random set includes $x$ or not:

$$
\begin{aligned}
E\left(X_{x}\right) & =K(x, x), \\
\operatorname{Cov}\left(X_{x}, X_{y}\right) & =\operatorname{det}\left(\begin{array}{ll}
K(x, x) & K(x, y) \\
K(y, x) & K(y, y)
\end{array}\right)-K(x, x) K(y, y) \\
& =-K(x, y) K(y, x) .
\end{aligned}
$$

If $K(x, y)=K(y, x)$, then the correlation is $\leq 0$, a distinctive feature of determinantal point processes.

(c) If the matrix $(K(x, y))_{x, y \in \mathcal{X}}$ has all real eigenvalues $\left\{\lambda_{x}\right\}_{x \in \mathcal{X}}$, many theorems become available for $N$, the total number of points in a realization of the process. These include:

- $N$ is distributed as a sum $\sum_{x \in \mathcal{X}} Y_{x}$ with $\left\{Y_{x}\right\}$ independent $0 / 1$ random variables having $P\left(Y_{x}=1\right)=\lambda_{x}$.

- Let $\mu$ and $\sigma$ denote the mean and variance of $N$. These are available in terms of $K$ from (b) above. Then the following refined form of the central limit theorem holds:

$$
\left|P\left\{\frac{N-\mu}{\sigma} \leq x\right\}-\frac{1}{\sqrt{2 \pi}} \int_{-\infty}^{x} e^{-t^{2} / 2} d t\right| \leq \frac{.80}{\sigma} .
$$

- The probability density $P(n)=P(N=n)$ is a Polya-frequency function. In particular the density is unimodal. Further, $\mid$ mode - mean $\mid \leq$ 1.

- There is a useful algorithm for simulating from $K$ (here assuming $K(x, y)=K(y, x))$.

Most of these properties follow from the fact that the generating function of $N$,

$$
E\left(x^{N}\right)=\operatorname{det}(I+(x-1) K)
$$

has all real zeros. See Pitman 60, or Hough, et al. [43, 44, for details.

(d) If $K_{n}$ is a sequence of such kernels converging to a kernel $K$, then, under mild restrictions, the associated point processes converge. See Soshnikov 
68] or Johansson and Nordenstam [48] for precise statements and remarkable examples. A simple example is given in Section 5 (Example 11).

A main result of our paper is that the carries and descent processes, and indeed any one-dependent point processes on the integers, are determinantal. The special case of carries is stated here; a proof of the general case is in Section 7

Theorem 3.1. The point process of carries and descents $P_{n}$ given in Section 2 is determinantal with correlation kernel $K(i, j)=k(j-i)$, where

$$
\sum_{m \in \mathbb{Z}} k(m) t^{m}=\frac{1}{1-(1-t)^{b}}
$$

Example 3. Take $b=2$. Then $\frac{1}{1-(1-t)^{2}}=\frac{1}{2 t(1-t / 2)}$. Replacing $t$ by $c t$ changes $k(m)$ to $k(m) c^{m}$ and $K(i, j)=k(j-i)$ to $c^{j-i} k(j-i)=c^{-i} K(i, j) c^{j}$. Conjugating $K$ by a diagonal matrix does not change determinants or the correlation functions. Thus setting $\frac{t}{2}=z$, the generating function becomes

$$
\frac{1}{4}\left(\frac{1}{z}+1+z+z^{2}+\cdots\right)
$$

When, e.g., $n-1=5$, the kernel is

$$
\left(\frac{1}{4}\right)\left[\begin{array}{lllll}
1 & 1 & 1 & 1 & 1 \\
1 & 1 & 1 & 1 & 1 \\
0 & 1 & 1 & 1 & 1 \\
0 & 0 & 1 & 1 & 1 \\
0 & 0 & 0 & 1 & 1
\end{array}\right]
$$

From this,

$$
\rho(A)= \begin{cases}0 & \text { if } A \text { has two consecutive entries } \\ \left(\frac{1}{4}\right)^{|A|} & \text { otherwise. }\end{cases}
$$

This is a manifestation of the one-dependence together with the fact that (for $b=2$ ), two consecutive carries are impossible. It follows that the possible configurations are all binary strings of length $n-1$ with no two consecutive 1's. This is a standard coding of the Fibonacci number $F_{n+1}$ (if $1,1,2,3,5$ are the first five Fibonacci numbers). For example, when $n=4$, the possible configurations are 000, 001, 010, 100, 101.

The eigenvalues of any finite matrix of the form (3.1) are real. This follows from the fact that these matrices are totally positive, see [29], and the well-known fact that the eigenvalues of a totally positive matrix are real (and nonnegative); see e.g. Corollary 6.6 of [1].

The classification of $[29$ also implies that the correlation kernels for $b \geq 3$ are not totally positive (for large enough matrix size), and we do not know if their eigenvalues are real.

Example 4. Take $b=3$. A straightforward expansion leads to

$$
\begin{aligned}
\sum_{m=-\infty}^{\infty} k(m) t^{m} & =\frac{1}{1-(1-t)^{3}}=\frac{1}{3 t\left(1-t+\frac{t^{2}}{3}\right)} \\
& =\frac{\left(1+t+\frac{2 t^{2}}{3}+\frac{t^{3}}{3}+\frac{t^{4}}{9}\right)}{3 t\left(1+\frac{t^{6}}{27}\right)} .
\end{aligned}
$$


Thus

- $k(n)=0$ for $n<-1$,

- $k(-1)=\frac{1}{3}$,

- $k(n)=0$ for $n=4+6 j, 0 \leq j<\infty$. For other $n \geq 2$,

- $k(n)=(-1)^{\left\lfloor\frac{n+1}{6}\right\rfloor}\left(\frac{1}{3}\right)^{\left\lfloor\frac{n+3}{2}\right\rfloor} 2^{\delta(n)}$, where $\delta(n)=1$ if $n=1 \bmod 6$ and 0 else.

For example, the first few values are:

\begin{tabular}{r|cccccccccccccccccc}
$m$ & -1 & 0 & 1 & 2 & 3 & 4 & 5 & 6 & 7 & 8 & 9 & 10 & 11 & 12 & 13 & 14 & 15 & 16 \\
\hline$k(m)$ & $\frac{1}{3}$ & $\frac{1}{3}$ & $\frac{2}{9}$ & $\frac{1}{9}$ & $\frac{1}{27}$ & 0 & $-\frac{1}{3^{4}}$ & $-\frac{1}{3^{4}}$ & $-\frac{2}{3^{5}}$ & $-\frac{1}{3^{5}}$ & $-\frac{1}{3^{6}}$ & 0 & $\frac{1}{3^{7}}$ & $\frac{1}{3^{7}}$ & $\frac{2}{3^{8}}$ & $\frac{1}{3^{8}}$ & $\frac{1}{3^{9}}$ & 0
\end{tabular}

It is amusing to see that the two-variable kernel $K(x, y)=k(y-x)$ reproduces the correlation functions found for this problem in Section 2 (Fact 4). Thus for $n=7$,

$$
(K(x, y))_{i, j=1}^{6}=\left[\begin{array}{cccccc}
1 / 3 & 2 / 9 & 1 / 9 & 1 / 27 & 0 & -1 / 81 \\
1 / 3 & 1 / 3 & 2 / 9 & 1 / 9 & 1 / 27 & 0 \\
0 & 1 / 3 & 1 / 3 & 2 / 9 & 1 / 9 & 1 / 27 \\
0 & 0 & 1 / 3 & 1 / 3 & 2 / 9 & 1 / 9 \\
0 & 0 & 0 & 1 / 3 & 1 / 3 & 2 / 9 \\
0 & 0 & 0 & 0 & 1 / 3 & 1 / 3
\end{array}\right] .
$$

\section{ONE-DEPENDENT PROCESSES}

Let $\left\{X_{i}\right\}_{i=0}^{N}$ be a binary stochastic process. Here $0<N \leq \infty$. The process is one-dependent if $\left\{X_{j}\right\}_{j=0}^{i-1},\left\{X_{j}\right\}_{j=i+1}^{N}$ are independent for all $i$. A natural example: let $\left\{Y_{i}\right\}_{i=0}^{N+1}$ be independent uniform random variables on $[0,1]$. Let $h:[0,1]^{2} \rightarrow\{0,1\}$ be a measurable function. Then $X_{i}=h\left(Y_{i}, Y_{i+1}\right), 0 \leq i \leq N$, is a one-dependent process called a two-block factor. Much of the literature on one-dependence is for stationary processes. However, if the $Y_{i}$ are independent but not identically distributed or $h=h_{i}$ depends on $i$, the associated $X_{i}$ is still onedependent. Our main results in Section 7 are for general one-dependent processes. We first describe some results for the stationary case.

Stationary one-dependent processes. A book-length review of this subject by de Valk [76] collects together many of the results, so we will be brief, focusing on later research. One of the first problems studied is the question of whether all one-dependent processes are two-block factors. Counterexamples were found 76 though it appears that most "natural" one-dependent processes are two-block factors. For example, let $f \in L^{2}(\pi)$ be a function on the unit circle. Let $K(i, j)=$ $\hat{f}(i-j), i, j \in \mathbb{Z}, \hat{f}$ the Fourier transform. Macchi [53] and Soshnikov [68] show that $K(i, j)$ is the kernel for a determinantal point process on $\mathbb{Z}$. Lyons and Steif [52] and Shirai and Takahashi 65, 66 develop remarkable properties of the associated point process. If $f(\theta)=a e^{-i \theta}+b+c e^{i \theta}$, the point process is one-dependent and [10] shows it is a two-block factor. See also [14.

A stationary one-dependent process is determined by the numbers $a_{i}=P\left(X_{1}=\right.$ $\left.X_{2}=\cdots=X_{i-1}=1\right), a_{1}=1$. Indeed, if $f=f_{1}, \ldots, f_{i-1} ; e=e_{i+1}, \ldots, e_{n-1}$ are binary strings, then $P(f, 0, e)+P(f, 1, e)=P(f) P(e)$. From this, $P(f, 0, e)=$ $P(f) P(e)-P(f, 1, e)$. By similar reductions, the probability of any pattern of occurrences can be reduced to a polynomial in the $a_{i}$. For example, $P(0,0,0)=$ $1-3 a_{2}+a_{2}^{2}+2 a_{3}-a_{4}$. There is a remarkable, simple formula for this polynomial as 
a minor of a Toeplitz matrix. We learned this from [61, Chap. 7]. A generalization to the nonstationary case is in Section 7

Theorem 4.1. For a stationary one-dependent process, let $a_{i}=P\left(X_{1}=X_{2}=\right.$ $\left.\cdots=X_{i-1}=1\right), a_{1}=1$. Let $t_{1}, \ldots, t_{n-1}$ be a binary string with $k$ zeros at positions $S=\left\{s_{1}<\cdots<s_{k}\right\} \subseteq[n-1]$. Then

$$
P\left(t_{1}, \ldots, t_{n-1}\right)=\operatorname{det}\left(a_{s_{j+1}-s_{i}}\right)_{i, j=0}^{k} .
$$

Here the determinant is of a $k+1$ by $k+1$ matrix, and one sets $s_{0}=0, s_{k+1}=$ $n, a_{0}=1$, and $a_{i}=0$ for $i<0$.

Proof. Theorem 4.1 follows from Proposition 2.2 and Theorem 4.2 in [61, Chap. 7], after elementary manipulations.

Remark. It follows from the discussion on [61, p. 140] that if all of the determinants (for all $n$ and subsets $S$ ) in Theorem 4.1 are nonnegative, then a stationary, onedependent process with these $a_{i}$ values exists.

Example 5. For any $n$, the sequence with $n-1$ ones has $k=0$ zeros. The relevant matrix is then 1 by 1 with entry $a_{n}$, giving this probability.

Example 6. To compute $P(0,0,0)$ one applies the theorem with $n=4$ and $S=$ $\{1,2,3\}$. It follows that

$$
P(0,0,0)=\operatorname{det}\left[\begin{array}{cccc}
1 & a_{2} & a_{3} & a_{4} \\
1 & 1 & a_{2} & a_{3} \\
0 & 1 & 1 & a_{2} \\
0 & 0 & 1 & 1
\end{array}\right]=1-3 a_{2}+a_{2}^{2}+2 a_{3}-a_{4}
$$

as above.

Our next result expresses the determinant in Theorem 4.1 as a skew-Schur function of ribbon (also called rim-hook) type. Some background on skew-Schur functions can be found in [54, Sec. 1.5]. For further references and recent work, one can consult [3] or [50. We will use the elementary symmetric functions $e_{r}$ in countably many variables defined as $e_{0}=1$ and

$$
e_{r}=\sum_{1 \leq i_{1}<i_{2}<\cdots<i_{r}} x_{i_{1}} x_{i_{2}} \cdots x_{i_{r}} .
$$

From [54, p. 20] these are algebraically independent, so there is a homomorphism of the ring of symmetric functions to $\mathbb{R}$ which sends each $e_{i}$ to $a_{i}$ from Theorem 4.1 .

Theorem 4.2. With notation as in Theorem 4.1, let $\lambda$ and $\mu$ be the partitions defined by

$$
\lambda_{i}=n-s_{i-1}-k+i-1, \mu_{i}=n-s_{i}-k+i-1, \quad 1 \leq i \leq k+1 .
$$

Let $\lambda^{\prime}, \mu^{\prime}$ denote the transposed partitions of $\lambda$ and $\mu$ and let $s_{\lambda^{\prime} / \mu^{\prime}}$ denote the corresponding skew-Schur function, obtained by specializing the elementary symmetric functions $e_{i}$ to equal $a_{i}$. Then

$$
P\left(t_{1}, \ldots, t_{n-1}\right)=s_{\lambda^{\prime} / \mu^{\prime}}
$$


Proof. First note that the $\lambda_{i}, \mu_{i}$ defined above are all nonnegative. From Macdonald [54, p. 71],

$$
s_{\lambda^{\prime} / \mu^{\prime}}=\operatorname{det}\left(e_{\lambda_{i}-\mu_{j}-i+j}\right)_{i, j=1}^{k+1},
$$

and $e_{r}$ is the $r$ th elementary symmetric function. When each $e_{r}$ is specialized to equal $a_{r}$, the quantity $s_{\lambda^{\prime} / \mu^{\prime}}$ becomes

$$
\operatorname{det}\left(a_{\lambda_{i}-\mu_{j}-i+j}\right)_{i, j=1}^{k+1}=\operatorname{det}\left(a_{s_{j}-s_{i-1}}\right)_{i, j=1}^{k+1},
$$

as desired.

One reason why Theorem 4.2 is interesting is that there are nonobvious equalities between ribbon skew-Schur functions. The paper [3] characterizes when two ribbon skew-Schur functions are equal; analogous results for more general skew-Schur functions are in [63. In particular, combining the results of [3] with Theorem 4.2 one immediately obtains the fact that a stationary one-dependent process is invariant under time reversal, i.e.

$$
P\left(t_{1}, \ldots, t_{n}\right)=P\left(t_{n}, \ldots, t_{1}\right)
$$

for all $t_{i}$. For another proof of invariance under time reversal, see [61, p. 139]. Peter Winkler notes that for the special case of the carries/descents example in Section 2 time reversal follows since replacing $B_{1}, \ldots, B_{n}$ by $b-B_{n}, \ldots, b-B_{1}$ reverses the descent set.

The determinantal formulae of Theorems 4.1 and 4.2 suggest that a determinantal point process is lurking nearby. This is indeed the case. In Section 7 (see Corollary (7.3) we prove the following.

Corollary 4.3. A stationary one-dependent process as in Theorem 4.1 is determinantal with kernel $K(x, y)=k(y-x)$ with

$$
\sum_{n \in \mathbb{Z}} k(n) z^{n}=-1 / \sum_{j=1}^{\infty} a_{j} z^{j} .
$$

A remarkable development, connecting stationary one-dependent processes to algebra appears in [61, Chap. 7]. They consider a graded algebra $A_{0} \oplus A_{1} \oplus A_{2}+\cdots$ with $A_{0}=k$, a ground field, and $A_{i} A_{j} \subseteq A_{i+j}$. For example, the space $k[x]$ of polynomials in one variable has $A_{0}=k$, and $A_{i}$ spanned by $x^{i}$. They assume that each $A_{i}$ is a finite-dimensional vector space of dimension $\operatorname{dim}\left(A_{i}\right)$. The algebra is quadratic if $A_{0}=k$, the algebra is generated by elements of $A_{1}$, and the relations defining the algebra are in $A_{2}$. For example, the commutative polynomial ring $k\left[x_{1}, \ldots, x_{n}\right]$ is generated by $x_{1}, \ldots, x_{n}$ and the quadratic relations $x_{i} x_{j}-x_{j} x_{i}=0$. Note that algebras need not be commutative.

A technical growth condition on the $\operatorname{dim}\left(A_{i}\right)$ which we will not explain here yields the Koszul algebras. These include many natural algebras occurring in mathematics (see 35] for a survey). As a simple example, consider the commutative polynomial ring generated by $x_{1}, x_{2}, \ldots, x_{n}$ with the additional relations $x_{i} x_{j}=0$ for $(i, j) \in E$, with $E$ the edge set of an undirected graph on $\{1,2, \ldots, n\}$ (loops allowed). This is Koszul [61, Chap. 2, Cor. 4.3]. Polishchuk and Positselski [61, Chap. 7, Cor. 4.3] prove the following remarkable result. 
Theorem 4.4. To every Koszul algebra A one can assign a stationary, one-dependent process via

$$
P\left(X_{1}=X_{2}=\cdots=X_{i-1}=1\right)=\frac{\operatorname{dim}\left(A_{i}\right)}{\operatorname{dim}\left(A_{1}\right)^{i}} \quad \text { for } i=1,2, \ldots
$$

Part of the reason that Theorem 4.4 is substantive is that the quantities $P\left(X_{1}=\right.$ $\left.X_{2}=\cdots=X_{i-1}=1\right)$ cannot assume arbitrary values; the determinants in Theorem 4.1 must be nonnegative.

Example 7. Consider the commutative polynomial algebra generated by $x_{1}, x_{2}$, $\ldots, x_{b}$ and the additional relations $x_{i}^{2}=0,1 \leq i \leq b$. The degree $i$ part $A_{i}$ is spanned by square-free monomials and has $\operatorname{dim}\left(A_{i}\right)=\left(\begin{array}{l}b \\ i\end{array}\right), 0 \leq i<\infty$. From Fact 2 of Section 2, we see that the associated one-dependent process is precisely the carries process of mod $b$ addition from our introduction. Note that since only the $\operatorname{dim}\left(A_{i}\right)$ matter, the algebra in this example can be replaced by the exterior algebra generated by $x_{1}, x_{2}, \ldots, x_{b}$ and the relations $x_{i} x_{j}=-x_{j} x_{i}$ for all $i, j$.

Example 8. Consider the commutative polynomial algebra generated by $x_{1}, x_{2}$, $\ldots, x_{b}$. The degree $i$ part $A_{i}$ is spanned by monomials of degree $i$ and has $\operatorname{dim}\left(A_{i}\right)=$ $\left(\begin{array}{c}b+i-1 \\ i\end{array}\right)$. From Example 2 of Section 2 the associated one-dependent process is precisely the complement of the carries process of $\bmod b$ addition. More generally, in Section 7, we show that the particle-hole involution of a (perhaps nonstationary) one-dependent process with respect to any subset is one-dependent.

Example 9. Consider the algebra

$$
k\left[x_{1}, \ldots, x_{n}\right] /\left\langle x_{i} x_{j}:(i, j) \in S \subset[1, n]^{2}\right\rangle,
$$

where the variables $x_{1}, \ldots, x_{n}$ do not commute. Here it is useful to think of $S$ as a directed graph with vertex set $\{1, \ldots, n\}$, loops allowed. From 61, p. 84], this algebra is Koszul. As noted in [61, Chap. 7, Prop. 5.1], the one-dependent process associated to this algebra can be described as follows: let $U_{1}, U_{2}, \ldots$ be independent, identically distributed in $\{1, \ldots, n\}$ and let $X_{i}=h\left(U_{i}, U_{i+1}\right)$, where $h(i, j)=0$ if $(i, j) \in S$ and $h(i, j)=1$ otherwise. Indeed, the dimensions satisfy $\operatorname{dim}\left(A_{0}\right)=1, \operatorname{dim}\left(A_{1}\right)=n, \operatorname{dim}\left(A_{i}\right)=$ number of paths of length $i-1$ in the complement of $S$, and so the chance of $i-1$ consecutive 1 's in the point process is equal to $\operatorname{dim}\left(A_{i}\right) / \operatorname{dim}\left(A_{1}\right)^{i}$.

The above argument shows that these algebras give rise to two-block-factor processes. In fact they are dense in the set of all two-block factors 61, Chap. 7, Prop. 5.2]. For a generalization of these processes, as well as a simple argument that they are determinantal, see Remark 5 after Theorem 5.3 .

Example 10. Consider $2 n$ points $x_{1}, x_{2}, \ldots, x_{2 n}$ in general position in projective space $\mathbb{P}^{n}$. The coordinate ring of this projective variety is known to be Koszul [61, p. 42] with $\operatorname{dim}\left(A_{0}\right)=1, \operatorname{dim}\left(A_{1}\right)=n+1, \operatorname{dim}\left(A_{i}\right)=2 n$ for $i \geq 2$. Thus $P\left(X_{1}=X_{2}=\cdots=X_{i-1}=1\right)=2 n /(n+1)^{i}$ defines a one-dependent process. When $n=1$, this is fair coin tossing. When $n=3$, it is the carries process for multiplying quaternions introduced in Section 6.

For general $n$, these one-dependent processes can be described as follows (and illustrate what one might consider to be a "probabilistic" description). Let $\{1,2$, $\ldots, n\}$ be ordered cyclically and let $*$ be another symbol. Choose $U_{i}$ i.i.d. in 
$\{*, 1, \ldots, n\}$. Let $X_{i}=h\left(U_{i}, U_{i+1}\right)$, where $h(*, x)=0$ for all $x, h(x, *)=1=$ $h(i, i+1)$ for $x \neq *$, and $h(i, j)=0$ otherwise. To see that this works, note that $P\left(X_{1}=1\right)=2 n /(n+1)^{2}$, since one has to choose $x$ different from $*$ first and then * or $x+1$ next. Similarly, for the chance of $i-11$ 's in a row, the first choice can be anything but $*$, then the next $i-2$ choices are determined and the last can be one of two.

We are certain that natural Koszul algebras will lead to natural point processes. We note further that [61, Sec. 7.6] shows how natural operations on algebras preserve the Koszul property. These include the operations of union and complements that we work with in Section 7 .

\section{Descents in PeRmutations}

A permutation $\sigma \in S_{n}$, the symmetric group, has a descent at $i$ if $\sigma(i)>\sigma(i+1)$. The set of such $i$ forms the descent set $D(\sigma)$. If the base $b$ in previous sections is large and $n$ stays fixed, a string of $n$ digits will have no repeated values and the descent theory of Section 2 and Section 3 above becomes the descent theory for random permutations. This is a venerable subject. Stanley [69, 71] reviews the basics. Some modern highlights are Solomon's descent algebra [67, the connections with the free Lie algebra [37, 64, Gessel's theory of enumerating permutations by descents and cycle structure 23, 38, quasi-symmetric functions and the theory of riffle shuffling [36, 73. Any Coxeter group has its own descent theory 67. There is some indication that arithmetic carries can be carried over [21.

This section introduces three examples where the descent set can be shown to be a one-dependent determinantal point process: uniform choice of permutations, nonuniform choice from the Mallows model, and independent trials. Then it shows that the closely related notion of the connectivity set of a permutation also yields a determinantal process. Finally, connections with binomial posets are mentioned.

Example 11 (The descent set of a uniformly chosen permutation). Consider the formula of Section 2, Fact 5 for the chance that a random $b$-ary string of length $n$ has descents exactly at $S \subseteq[n-1]$. Passing to the limit, as $b \nearrow \infty$ using $\left(\begin{array}{c}a+b-1 \\ b-1\end{array}\right) \sim \frac{b^{a}}{a !}$ gives a classical formula for the chance that a uniformly chosen $\sigma \in S_{n}$ has descents exactly at $S$ :

$$
P_{n}(D(\sigma)=S)=\operatorname{det}\left[1 /\left(s_{j+1}-s_{i}\right) !\right] .
$$

For $S=1 \leq s_{1}<s_{2}<\cdots<s_{k} \leq n-1$, the determinant is of a $(k+1) \times(k+1)$ matrix with $(i, j)$ entry $1 /\left(s_{j+1}-s_{i}\right) !,(i, j) \in[0, k] \times[0, k]$ and $s_{0}=0, s_{k+1}=n$. This formula is originally due to MacMahon [55]. See Stanley [70, p. 69] or Gessel and Viennot [39] for modern proofs.

Equation (5.1) allows us to see that the descent set of a uniformly random permutation, treated as a point process, is determinantal. The proof follows from (5.1) and Corollary 7.6 in Section 7.

Theorem 5.1. There exists a stationary point process on $\mathbb{Z}$, call it $P$, such that its restriction to any interval of length $n-1$ coincides with $P_{n}$ of (5.1). The process $P$ is one-dependent and determinantal. Its correlation kernel $K(x, y)=k(y-x)$ with

$$
\sum_{m \in \mathbb{Z}} k(m) z^{m}=\frac{1}{1-e^{z}}
$$


Remarks.

(1) Theorem 5.1 can also be seen by passing to the limit in Theorem 3.1. Replacing $t$ in the generating function $1 /\left(1-(1-t)^{b}\right)$ by $-z / b$ and letting $b \nearrow \infty$ gives (5.2).

(2) The Bernoulli numbers $B_{n}$ are defined by $z /\left(e^{z}-1\right)=\sum_{n=0}^{\infty} B_{n} z^{n} / n$ ! These are very well-studied. It is known that $B_{2 i+1}=0$ for $i \geq 1$, $B_{0}=1, B_{1}=-\frac{1}{2}, B_{2}=\frac{1}{6}, B_{4}=-\frac{1}{30}, B_{6}=\frac{1}{42}, \ldots$ We see that $k(m)=$ $-B_{m+1} /(m+1)$ !.

(3) From these calculations, the kernel $K$ is

$$
K=\left[\begin{array}{ccccc}
\frac{1}{2} & -\frac{1}{12} & 0 & \frac{1}{720} & \cdots \\
-1 & \frac{1}{2} & -\frac{1}{12} & 0 & \ddots \\
& -1 & \ddots & \ddots & \ddots \\
& & \ddots & \ddots & \ddots \\
& & & \ddots & \ddots
\end{array}\right] .
$$

(4) The correlation functions of $P$ (and $P_{n}$ ) can be computed explicitly by passage to the limit from Fact 5 of Section 2. Let $A=A_{1} \cup A_{2} \cup \cdots \cup A_{k}$ be a decomposition of the finite set $A \subseteq \mathbb{Z}$ into disjoint blocks of adjacent integers. If $\left|A_{i}\right|=a_{i}$,

$$
\rho(A)=\prod_{i=1}^{k} \frac{1}{\left(a_{i}+1\right) !} .
$$

(5) For any $n=1,2, \ldots$, let $K_{n}(i, j)=k(j-i)$ be the $n \times n$ top left corner block of $K$. Let $d(\pi)$ denote the number of descents of $\pi$ and let

$$
A_{n+1}=\sum_{\pi \in S_{n+1}} x^{d(\pi)}
$$

be the $(n+1)$ st Eulerian polynomial (see, e.g., [70, p. 22]). Then item (c) preceding Theorem 3.1 yields that

$$
A_{n+1}(x)=\operatorname{det}\left(I+(x-1) K_{n}\right)(n+1) !
$$

It is known [15, p. 292] that $A_{n+1}(x)$ has all real zeros $\alpha_{1}, \alpha_{2}, \ldots, \alpha_{n}$. From the development in Section $3, N_{n+1}$, the number of descents in a random permutation from $S_{n+1}$, is the sum of $n$ independent Bernoulli random variables with success rates $\left(1-\alpha_{j}\right)^{-1}, 1 \leq j \leq n$. One easily computes

$$
\begin{aligned}
E\left(N_{n+1}\right)=\operatorname{tr}\left(K_{n}\right)=\frac{n}{2}, \quad \operatorname{Var}\left(N_{n+1}\right) & =\operatorname{tr}\left(K_{n}-K_{n}^{2}\right) \\
& =\frac{n}{2}-\left(\frac{5 n}{12}-\frac{1}{6}\right)=\frac{n+2}{12} .
\end{aligned}
$$

It follows by Harper's method (41, 60] ) that one has a central limit theorem:

$$
\frac{N_{n+1}-\frac{n}{2}}{\sqrt{\frac{n+2}{12}}} \Longrightarrow \mathcal{N}(0,1) \text {. }
$$


(6) Chebikin 12 studied the "alternating descent set" $A(\sigma)$ of a random permutation, defined as the set of positions at which the permutation has an alternating descent, that is, an ordinary descent if $i$ is odd, or an ascent if $i$ is even. Combining Lemma 2.3.1 of [12] with the argument used to prove (5.1), one obtains that for $|S|=k$,

$$
P_{n}(A(\sigma)=S)=\operatorname{det}\left[E_{s_{j+1}-s_{i}} /\left(s_{j+1}-s_{i}\right) !\right]_{i, j=0}^{k},
$$

where $E_{n}$ is the $n$th Euler number defined by $\sum_{n \geq 0} E_{n} z^{n} / n !=\tan (z)+$ $\sec (z)$, and $s_{0}=0, s_{k+1}=n$. Applying our Theorem 7.5 in Section 7, it follows that $P_{n}$ is obtained by restricting to any interval of length $n-1$ the stationary, one-dependent, determinantal process with correlation kernel $K(x, y)=k(y-x)$ with

$$
\sum_{m \in \mathbb{Z}} k(m) z^{m}=\frac{1}{1-(\tan (z)+\sec (z))^{-1}} .
$$

(7) Modern combinatorics suggests a host of potential generalizations of Theorem [5.1. Let $P$ be a partial order on $[n]$. A linear extension of $P$ is a permutation $\sigma \in S_{n}$ such that if $i$ is less than $j$ according to $P$, then $\sigma(i)<\sigma(j)$. Let $\mathcal{L}(P)$ denote the set of linear extensions of $P$. For each $\sigma \in \mathcal{L}(P)$, let

$$
\begin{aligned}
D(\sigma) & =\{i: \sigma(i)>\sigma(i+1)\}, & \text { the descent set of } \sigma, \\
\wedge(\sigma) & =\{i: \sigma(i-1)<\sigma(i)>\sigma(i+1)\}, & \text { the peak set of } \sigma .
\end{aligned}
$$

Choosing $\sigma$ uniformly in $\mathcal{L}(P)$ gives two point processes. This section has focused on ordinary descents; that is, $P$ is the trivial poset with no restrictions. There are many indications that "natural" posets will give rise to determinantal point processes. For background and first results, see Brenti [9] or Stembridge [75].

(8) Finally, we mention that it would be interesting to find analogs of Theorem 5.1 for other Coxeter groups. For the hyperoctahedral group $B_{n}$ consisting of the $2^{n} n$ ! signed permutations, descents can be defined using the linear ordering

$$
1<2<\cdots<n<-n<\cdots<-2<-1 .
$$

We say that

(a) $\sigma$ has a descent at position $i(1 \leq i \leq n-1)$ if $\sigma(i)>\sigma(i+1)$;

(b) $\sigma$ has a descent at position $n$ if $\sigma(n)<0$.

For example, $-4-132-5 \in B_{5}$ has descent set $\{2,3,5\}$. Reiner 62] shows that the chance that a random element of $B_{n}$ has descent set $\left\{s_{1}<\cdots<s_{r}\right\} \subseteq\{1,2, \ldots, n\}$ is $\operatorname{det}(a(i, j))_{i, j=0}^{r}$, where

$$
a(i, j)= \begin{cases}\frac{1}{\left(s_{j+1}-s_{i}\right) !} & \text { if } 0 \leq j \leq r-1, \\ \frac{1}{2^{n-s_{i}}\left(n-s_{i}\right) !} & \text { if } j=r .\end{cases}
$$

Here $s_{0}=0$. Theorem 7.5 in Section 7 shows that the resulting processes are determinantal (but not stationary).

Example 12 (Descents from nonuniform distributions on permutations). In a variety of applications, nonuniform distributions are used on permutations. A widely 
used model is

$$
P_{\theta}(\sigma)=Z^{-1} \theta^{d\left(\sigma, \sigma_{0}\right)}, \quad \sigma \in S_{n} .
$$

Here, $0<\theta \leq 1$ is a fixed concentration parameter, $\sigma_{0} \in S_{n}$ is a fixed location parameter, $d\left(\sigma, \sigma_{0}\right)$ is a metric on $S_{n}$ and $Z=\sum_{\sigma} \theta^{d\left(\sigma, \sigma_{0}\right)}$ is the normalizing constant. These are called "Mallows models through the metric $d$." When $\theta=1$, $P_{1}$ is the uniform distribution. For $0<\theta<1, P_{\theta}$ is largest at $\sigma_{0}$ and falls off as $\sigma$ moves away from $\sigma_{0}$. See [16], 24] or [57] for background and references.

Perhaps the most widely used metric is

$$
\begin{aligned}
d\left(\sigma, \sigma_{0}\right)= & \text { minimum number of pairwise adjacent transpositions } \\
& \quad \text { to bring } \sigma \text { to } \sigma_{0}, \\
= & I\left(\sigma \sigma_{0}^{-1}\right), \text { the number of inversions in } \sigma \sigma_{0}^{-1} .
\end{aligned}
$$

This is called "Kendall's tau" in the statistics literature. References, extensions and properties are in [20, Sect. 4], [32, [56]. We mention that the normalizing constant of the Mallows model through Kendall's tau is given by $Z=\prod_{i=1}^{n} \frac{\theta^{i}-1}{\theta-1}$ [70, p. 21].

With $\theta, \sigma_{0}$ and $d$ fixed, one may ask any of the usual questions of applied probability and enumerative combinatorics: "Picking a permutation randomly from $P_{\theta}(\cdot)$, what is the distribution of the cycle structure, longest increasing subsequence, ...?" For general metrics, these are open research problems. Even the algorithmic task of sampling from $P_{\theta}$ leads to difficult problems. See [22] or [20].

For the Mallows model $P_{\theta}$ through Kendall's tau (5.4) and $\sigma_{0}=$ id, we show that the descent set of $\sigma, D(\sigma)=\{i: \sigma(i+1)<\sigma(i)\}$ forms a determinantal point process with simple properties. In what follows we use the $q$-analog notation that $i_{q}=\frac{q^{i}-1}{q-1}$ and $n !_{q}=\prod_{i=1}^{n} i_{q}$, and take $\theta=q$ as is conventional in combinatorial work in the subject.

Proposition 5.2. Let $P_{q}(0<q<1)$ be the Mallows model through Kendall's tau (5.3), (5.4) on $S_{n}$.

(a) The chance that a random permutation chosen from $P_{\theta}$ has descent set $s_{1}<s_{2}<\cdots<s_{k}$ is

$$
\operatorname{det}\left[\frac{1}{\left(s_{j+1}-s_{i}\right) !_{q}}\right]_{i, j=0}^{k}
$$

with $s_{0}=0$ and $s_{k+1}=n$.

(b) The point process associated to $D(\sigma)$ is stationary, one-dependent, and determinantal with kernel $K(x, y)=k(y-x)$, where

$$
\sum_{m \in \mathbb{Z}} k(m) z^{m}=\frac{1}{1-\left(\sum_{m=0}^{\infty} z^{m} / m !_{q}\right)^{-1}} .
$$

(c) The chance of finding $k$ descents in a row is $q^{\left(\begin{array}{c}k+1 \\ 2\end{array}\right)} /(k+1) !_{q}$. In particular, the chance of a descent at any given location is $q /(q+1)$. The number of descents has mean $\mu(n, q)=\frac{(n-1) q}{q+1}$ and variance $q\left[\frac{\left(q^{2}-q+1\right) n-q^{2}+3 q-1}{\left(q^{2}+q+1\right)(q+1)^{2}}\right]$. Normalized by its mean and variance, the number of descents has a limiting standard normal distribution.

Proof. Part (a) of Proposition 5.2 follows from Stanley [70, Ex. 2.2.5]. Part (b) follows from Corollary 7.6 in Section 7 and elementary calculations. To compute 
the chance of $k$ consecutive descents, note by stationarity that this is the chance of the permutation $\sigma(i)=k+2-i$ in $S_{k+1}$ under the $P_{q}$ measure on $S_{k+1}$. This

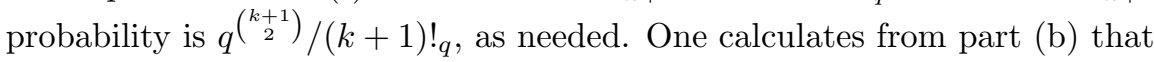

$$
k(-1)=1, k(0)=\frac{q}{q+1}, k(1)=\frac{q^{2}}{(q+1)^{2}\left(q^{2}+q+1\right)} .
$$

Recall from the remarks preceding Theorem 3.1 that

$$
E\left(x^{N}\right)=\operatorname{det}(I+(x-1) K),
$$

where $N$ is the total number of particles. Thus

$$
\begin{gathered}
E(|D(\sigma)|)=\operatorname{tr}\left(K_{n-1}\right)=(n-1) k(0), \\
\operatorname{Var}(|D(\sigma)|)=\operatorname{tr}\left(K_{n-1}-K_{n-1}^{2}\right)=(n-1) k(0)-(n-1) k(0)^{2}-2(n-2) k(1) k(-1),
\end{gathered}
$$

and the proof of part (c) is complete.

Remarks.

(1) From part (c), the correlation functions are given explicitly, just as in remark 4 following Theorem 5.1 .

(2) It would be interesting to have a direct combinatorial proof of the onedependence in Proposition 5.2

Example 13 (Descents for independent trials). The carries in the carries process have an equivalent description in terms of descents in an independent and uniform sequence. In this example we generalize to descents in a sequence $Y_{1}, Y_{2}, \ldots, Y_{n}$ with $Y_{i}$ independent and identically distributed with $P\left(Y_{1}=j\right)=p_{j}$. Here $0 \leq j \leq b-1$ to match previous sections (and of course $0 \leq p_{j} \leq 1, \sum p_{j}=1$ ), but in fact $b=\infty$ can be allowed. The descents are related to the order statistics for discrete random variables 2 , 30, but we have not seen this problem previously treated in the probability or statistics literature. Of course, if the $Y_{i}$ are independent and identically distributed from a continuous distribution, the descent theory is the same as the descent theory for a random permutation.

It is useful to have the complete homogeneous symmetric functions

$$
h_{k}\left(x_{1}, x_{2}, \ldots, x_{m}\right)=\sum_{1 \leq i_{1} \leq \cdots \leq i_{k} \leq m} x_{i_{1}} x_{i_{2}} \cdots x_{i_{k}} .
$$

Theorem 5.3. If $Y_{1}, Y_{2}, \ldots, Y_{n}$ are independent with common probability $P\left(Y_{1}=\right.$ $j)=p_{j}, 0 \leq j \leq b-1$, the descents in $Y_{1}, Y_{2}, \ldots, Y_{n}$ form a stationary onedependent point process on $[n-1]$ with

$$
P_{n}(S)=\operatorname{det}\left(h_{s_{j+1}-s_{i}}\right)_{0 \leq i, j \leq k} \quad \text { with } s_{0}=0, s_{k+1}=n,
$$

for $S=\left\{s_{1}<s_{2}<\cdots<s_{k}\right\}$. The $h_{j}$ are evaluated at $p_{0}, \ldots, p_{b-1}$. Moreover, this process is determinantal with $K(i, j)=k(j-i)$ and $\sum_{l=-\infty}^{\infty} k(l) z^{l}=\frac{1}{1-\prod_{i=0}^{b-1}\left(1-p_{i} z\right)}$.

Proof. Let $\alpha_{n}(S)=P_{n}\left(\operatorname{des}\left(Y_{1}, Y_{2}, \ldots, Y_{n}\right) \subseteq S\right)=h_{s_{1}} h_{s_{2}-s_{1}} \cdots h_{n-s_{k}}$. The determinant formula follows from the argument for Fact 5 in Section 2. Now, Corollary 7.6 implies that $\hat{k}(z)=\frac{1}{1-\hat{e}(z)^{-1}}$, with $\hat{e}(z)=\sum_{l=0}^{\infty} h_{l} z^{l}=\prod_{i=0}^{b-1}\left(1-p_{i} z\right)^{-1}$ from, e.g., Macdonald [54, p. 21]. 
Remarks.

(1) Setting $p_{i}=1 / b, \hat{k}(z)=\frac{1}{1-(1-z / b)^{b}}$, this is equivalent to our result in Theorem 3.1. by changing variable $z$ to $z / b \rightarrow t$, which doesn't change correlations.

(2) From the theorem, the associated point process is one-dependent. If $X_{i}=1$ or 0 depending on whether there is a descent at $i$ or not, then clearly

$$
P\left(X_{1}=X_{2}=\cdots=X_{i}=1\right)=e_{i+1}\left(p_{0}, \ldots, p_{b-1}\right),
$$

where $e_{r}\left(x_{1}, \ldots, x_{m}\right)=\sum_{1 \leq i_{1}<\cdots<i_{r} \leq m} x_{i_{1}} x_{i_{2}} \cdots x_{i_{m}}$ is the $r$ th elementary symmetric function. For example,

$$
\begin{gathered}
P\left(X_{i}=1\right)=\frac{1}{2}-\frac{1}{2} \sum_{i} p_{i}^{2}, \\
P\left(X_{i}=X_{i+1}=1\right)=\frac{1}{6}-\frac{1}{2} \sum_{i} p_{i}^{2}+\frac{1}{3} \sum_{i} p_{i}^{3} .
\end{gathered}
$$

This allows a straightforward formulation (and proof) of the central limit theorem for the number of descents. As in Section 2, simple expressions for the correlation functions are available in terms of $P\left(X_{1}=X_{2}=\cdots=\right.$ $\left.X_{i}=1\right)$.

(3) As we show in Corollary 7.7, one can express $P_{n}(S)$ as a skew-Schur function of ribbon type. For special choices of the $p_{i}$ 's, this symmetric function interpretation may facilitate computation. For example, [54, Sect. 1.5] gives $s_{\lambda / \mu}=\sum_{\nu} c_{\mu \nu}^{\lambda} s_{\nu}$ with $c_{\mu \nu}^{\lambda}$ the Littlewood-Richardson coefficients and $s_{\nu}$ the Schur function. If $p_{i}=z q^{i}(i=0,1, \ldots, b-1, z$ a normalizing constant $)$, then $s_{\nu}$ is nonzero only if $\nu$ has length $\leq b$, in which case,

$$
s_{\nu}=z^{|\nu|} q^{n(\nu)} \prod_{x \in \nu} \frac{1-q^{b+c(x)}}{1-q^{h(x)}} ;
$$

see Macdonald [54, p. 44] for an explanation of the notation.

(4) Coming back to carries, if a column of numbers is chosen independently on $\{0,1, \ldots, b-1\}$ from a common distribution $\left\{p_{i}\right\}_{i=0}^{b-1}$, the remainder column after addition evolves as a Markov chain with starting distribution $\left\{p_{i}\right\}$ and transition matrix $P(i, j)=p_{j-i}($ indices $\bmod b)$. This is a circulant with known eigenvalues and eigenvectors. We do not know the distribution of carries.

(5) There is a sweeping generalization of this example which leads to a large collection of determinantal point processes. Let $R$ be an arbitrary subset of $[N] \times[N]$. Fix a probability distribution $\theta_{1}, \theta_{2}, \ldots, \theta_{N}$ on $[N]$. A point process $P_{n}$ on $[n-1]$ arises as follows: pick $Y_{1}, Y_{2}, \ldots, Y_{n}$ independently from $\left\{\theta_{i}\right\}$. Let

$$
X_{i}=\left\{\begin{array}{ll}
1 & \text { if }\left(Y_{i}, Y_{i+1}\right) \notin R \\
0 & \text { otherwise }
\end{array}, 1 \leq i \leq n-1 .\right.
$$

Let $S=\left\{i: X_{i}=1\right\}$. Using essentially the arguments above, it is proved in Brenti [9, Thm. 7.32] that

$$
P_{n}(S)=\operatorname{det}\left[h_{s_{j+1}-s_{i}}^{R}\right]_{i, j=0}^{k} \quad \text { for } S=\left\{s_{1}<\cdots<s_{k}\right\}, s_{0}=0, s_{k+1}=n \text {, }
$$


where $h_{j}^{R}=h_{j}^{R}\left(\theta_{1}, \ldots, \theta_{N}\right):=P_{j}(\emptyset)$. Here, as usual, $h_{0}^{R}=1, h_{j}^{R}=0$ for $j<0$. This falls into the domain of Theorem 7.5 and its corollary. It follows that $P_{n}$ is determinantal with kernel $K(i, j)=k(j-i), \quad \sum_{l \in \mathbb{Z}} k(l) z^{l}=$ $\frac{1}{\left(1-\left(\sum_{j=0}^{\infty} h_{j}^{R} z^{j}\right)^{-1}\right)}$.

Our examples are the special case

$$
R(i, j)= \begin{cases}1 & \text { if } i \leq j, \\ 0 & \text { otherwise }\end{cases}
$$

We are certain that there are many other interesting cases.

Example 14 (Connectivity set of a permutation). Stanley [74] studied a "dual" notion to the descent set of a permutation, which he called the connectivity set. For $\sigma \in S_{n}$, the connectivity set $C(\sigma)$ is defined as the set of $i, 1 \leq i \leq n-1$, such that $\sigma \in S_{1, \ldots, i} \times S_{i+1, \ldots, n}$. For example, the permutation $\sigma=312546$ satisfies $C(\sigma)=\{3,5\}$. The connectivity set also arises in the analysis of quicksort, where it is called the set of splitters of a permutation [77, Sec. 2.2].

We will prove that the connectivity set of a random element of $S_{n}$ is a determinantal point process and determine its correlation kernel. For this the following two facts are helpful:

(1) A permutation $\sigma \in S_{n}$ is called indecomposable or connected if $C(\sigma)=$ $\emptyset$. Comtet [15, Exer. VII.16] shows that the number $f(n)$ of connected permutations in $S_{n}$ satisfies

$$
\sum_{n \geq 1} f(n) x^{n}=1-\frac{1}{\sum_{n \geq 0} n ! x^{n}} .
$$

(An asymptotic expansion of $f(n)$ is given in [15, Exer. VII.17]).

(2) [74, Prop. 1.1] Letting $S=\left\{s_{1}<s_{2}<\cdots<s_{k}\right\}$ be a subset of $\{1,2, \ldots$, $n-1\}$,

$$
\left|\left\{\sigma \in S_{n}: S \subseteq C(\sigma)\right\}\right|=s_{1} !\left(s_{2}-s_{1}\right) ! \cdots\left(s_{k}-s_{k-1}\right) !\left(n-s_{k}\right) ! .
$$

The following is our main result.

Theorem 5.4. Let $C(\sigma)$ denote the connectivity set of a permutation $\sigma$ chosen uniformly at random from $S_{n}$. The point process corresponding to $C(\sigma) \cup\{0, n\}$ is determinantal, with state space $\{0, \ldots, n\}$ and correlation kernel $K(x, y)$ satisfying

$$
\begin{aligned}
& K(0, y)=1 \quad \text { all } y, \\
& K(n, y)=\delta_{n, y} \quad \text { all } y, \\
& K(x, y)=1 /\left(\begin{array}{l}
n \\
x
\end{array}\right) \quad 0, n \neq x \leq y, \\
& K(x, y)=1 /\left(\begin{array}{l}
n \\
x
\end{array}\right)-1 /\left(\begin{array}{l}
n-y \\
n-x
\end{array}\right) \quad 0, n \neq x>y .
\end{aligned}
$$

Note that in the statement of the theorem, $0, n$ are always points of the process.

Proof. The first step is to observe that $C(\sigma) \cup\{0, n\}$ can be obtained as a trajectory of a certain Markov chain, starting at 0 , with transition probabilities

$$
P(i, j)=\frac{(n-j) ! f(j-i)}{(n-i) !} .
$$

Here we take $f(0)=0$, so $P(i, i)=0$ for all $i$. To prove (5.7), note that $C(\sigma)=$ $\left\{i_{1}, \ldots, i_{k}\right\}$ if and only if the following events $E_{1}, \ldots, E_{k+1}$ occur: 
- $E_{1}:\left\{\pi(1), \ldots, \pi\left(i_{1}\right)\right\}=\left\{1, \ldots, i_{1}\right\}$ and $\pi$ restricted to $\left\{1, \ldots, i_{1}\right\}$ is indecomposable.

- $E_{2}:\left\{\pi\left(i_{1}+1\right), \ldots, \pi\left(i_{2}\right)\right\}=\left\{i_{1}+1, \ldots, i_{2}\right\}$ and $\pi$ restricted to $\left\{i_{1}+\right.$ $\left.1, \ldots, i_{2}\right\}$ is indecomposable.

- $\cdots$

- $E_{k+1}:\left\{\pi\left(i_{k}+1\right), \ldots, \pi(n)\right\}=\left\{i_{k}+1, \ldots, n\right\}$ and $\pi$ restricted to $\left\{i_{k}+\right.$ $1, \ldots, n\}$ is indecomposable.

Letting $f(n)$ denote the number of indecomposable permutations in $S_{n}$, the probability of $E_{1}$ is clearly $\frac{f\left(i_{1}\right)\left(n-i_{1}\right) !}{n !}$. The probability of $E_{2}$ given $E_{1}$ is $\frac{f\left(i_{2}-i_{1}\right)\left(n-i_{2}\right) !}{\left(n-i_{1}\right) !}$ and the probability of $E_{3}$ given $E_{1}, E_{2}$ is $\frac{f\left(i_{3}-i_{2}\right)\left(n-i_{3}\right) !}{\left(n-i_{2}\right) !}$, etc., as claimed.

Now [4, Thm. 1.1] implies that the point process on $C(\sigma) \cup\{0, n\}$ is determinantal with correlation kernel

$$
K(x, y)=\delta_{0, x}+Q(0, x)-Q(y, x)
$$

where

$$
Q=P+P^{2}+P^{3}+\cdots .
$$

To compute $Q$, let $\left[x^{r}\right] g(x)$ denote the coefficient of $x^{r}$ in a series $g(x)$, and note that

$$
\begin{aligned}
P^{2}(i, j) & =\sum_{l} P(i, l) P(l, j) \\
& =\sum_{l} \frac{(n-l) ! f(l-i)}{(n-i) !} \frac{(n-j) ! f(j-l)}{(n-l) !} \\
& =\frac{(n-j) !}{(n-i) !}\left[x^{j-i}\right]\left(1-\frac{1}{\sum_{n \geq 0} n ! x^{n}}\right)^{2} .
\end{aligned}
$$

The last equality used (5.5). A similar computation shows that

$$
P^{r}(i, j)=\frac{(n-j) !}{(n-i) !}\left[x^{j-i}\right]\left(1-\frac{1}{\sum_{n \geq 0} n ! x^{n}}\right)^{r},
$$

and thus

$$
\begin{aligned}
Q(i, j) & =\frac{(n-j) !}{(n-i) !}\left[x^{j-i}\right] \frac{\left(1-\frac{1}{\sum_{n \geq 0} n ! x^{n}}\right)}{\left(\sum_{n \geq 0} n ! x^{n}\right)^{-1}} \\
& =\frac{(n-j) !}{(n-i) !}\left[x^{j-i}\right]\left(\sum_{n \geq 1} n ! x^{n}\right) .
\end{aligned}
$$

Thus $Q(i, j)=1 /\left(\begin{array}{c}n-i \\ n-j\end{array}\right)$ if $i<j$, and is 0 otherwise. The theorem follows from this and (5.8).

Remarks. (1) The connectivity set $C(\sigma)$ is a simple example of a determinantal process that is not one-dependent. Indeed, from (5.6), $P(1 \in C(\sigma))=$ $\frac{1 !(n-1) !}{n !}, P(3 \in C(\sigma))=\frac{3 !(n-3) !}{n !}$, and $P(1,3 \in C(\sigma))=\frac{1 ! 2 !(n-3) !}{n !} \neq P(1 \in$ $C(\sigma)) P(3 \in C(\sigma))$. 
(2) Unlike the other point processes considered in this paper, the expected number of points tends to 0 as $n \rightarrow \infty$. Indeed, applying (5.6) gives that

$$
E|C(\sigma)|=\sum_{i=1}^{n-1} P(i \in C(\sigma))=\sum_{i=1}^{n-1} \frac{1}{\left(\begin{array}{c}
n \\
i
\end{array}\right)} \rightarrow 0
$$

as $n \rightarrow \infty$.

Binomial posets. In Doubilet-Rota-Stanley [25, binomial posets were introduced as a unifying mechanism to "explain" the many forms of generating functions that appear in enumerative combinatorics. Briefly, $\mathcal{X}$ is a binomial poset if for every interval $[x, y]$, the length of all maximal chains is the same (say $n_{x y}$ ) and the number of maximal chains in an interval of length $n$ does not depend on the particular interval. This number $B(n)$ is called the factorial function. For the usual Boolean algebra of subsets, $B(n)=n$ !. For the subspaces of a vector space over a finite field, $B(n)=n !_{q}$ ( $q$-factorial). There are many further examples.

Stanley [69] has shown that many enumerative formulae generalize neatly to the setting of binomial posets. Some of these developments give new determinantal point processes. Here is an example.

Example 15 (Union of descent sets).

Theorem 5.5. Pick $\sigma_{1}, \sigma_{2}, \ldots, \sigma_{n}$ independently from the Mallows model through Kendall's tau (5.3) on the symmetric group $S_{n}$. Let $S=\left\{s_{1}<\cdots<s_{k}\right\}$ be the union of their descent sets. Then

$$
P_{n}(S)=\operatorname{det}\left[\frac{1}{\left(\left(s_{j+1}-s_{i}\right) !_{q}\right)^{r}}\right],
$$

where $s_{0}=0, s_{k+1}=n$. The associated point process is stationary, one-dependent, and determinantal with $K(x, y)=k(y-x)$ for $\hat{k}(z)=\frac{1}{1-1 / \hat{e}(z)}$, where $\hat{e}(z)=$ $\sum_{l=0}^{\infty} z^{l} /\left(l !_{q}\right)^{r}$.

Proof. The formula for $P_{n}(S)$ is Corollary 3.2 of [69], so the theorem follows from one of our main results proved in Section 7 (Corollary 7.6).

When $q=1$ and $r=2, \hat{e}(z)=I_{0}(2 \sqrt{z})$, the classical modified Bessel function. Feller [31, §II.7] develops a host of connections with stochastic processes.

Remark. The intersection of two independent point processes with correlation functions $\rho^{1}(A), \rho^{2}(A)$ is a point process with correlation function $\rho^{1}(A) \rho^{2}(A)$. If both processes are determinantal, this is a product of determinants, but in general the intersection or union of determinantal processes is not determinantal. In Subsection 7.1, we show that all one-dependent processes are determinantal. Since the intersection of descent sets of independent permutations is one-dependent, and taking complements preserves one-dependence, this gives another proof that the union process in Theorem 5.5 is determinantal.

We have not pursued other examples but again believe there is much else to be discovered. 


\section{More General CARries}

Consider the quaternions $Q_{8}=\{ \pm 1, \pm i, \pm j, \pm k\}$. The center $Z$ of $Q_{8}$ is $\{ \pm 1\}$. Choose coset representatives $X=\{1, i, j, k\}$ for $Z$ in $Q_{8}$ so any element can be uniquely represented as $g=z x$. The coset representatives are multiplied by the familiar rule $k \curvearrowright i \curvearrowright j \curvearrowright k$ so $i j=k$ and $k j=-i$, etc. If we multiply $g_{1} g_{2} \cdots g_{k}=$ $\left(z_{1} x_{1}\right)\left(z_{2} x_{2}\right) \cdots\left(z_{k} x_{k}\right)$, the $z_{i} \in\{ \pm 1\}$ can all be moved to the left and we must multiply $x_{1} x_{2} \cdots x_{k}$, keeping track of the "carries", here \pm 1 . Evidently, if $\left\{g_{i}\right\}$ are chosen uniformly at random in $Q_{8}$, both $\left\{z_{i}\right\}$ and $\left\{x_{i}\right\}$ are independent and uniform in $Z$ and $X$. Thus we have the following problem: choose $X_{1}, X_{2}, \ldots, X_{k}$ uniformly in $X$ and multiply as $X_{1}, X_{1} X_{2},\left(X_{1} X_{2}\right) X_{3}, \ldots$,

$$
\begin{gathered}
X_{1} \\
X_{2} \\
X_{3} \\
\vdots \\
X_{k} .
\end{gathered}
$$

This gives a process of remainders and carries as in Section 1 .

\section{Example 16.}

$$
\begin{array}{ccc}
k & \cdot & k \\
k & & 1 \\
i & \cdot & \\
i & & 1 \\
k & \cdot & k \\
j & \cdot & i
\end{array} \quad \text { gives }(-1)^{5} k=-k .
$$

It is almost obvious that the carries form a stationary, one-dependent, two-block process with $P(i-1$ ones in a row $)=\frac{6}{4^{i}}, 2 \leq i<\infty$. Further, the remainders in the second column are independent and uniform on $\{1, i, j, k\}$ and there is a simple "descent" rule which determines the joint law of the dots (Example 18 below).

We also mention, by comparison with Example 10 in Section 4, that the carries process for the quaternions is the same point process that arises from the coordinate ring of 6 generic points in projective space $\mathbb{P}^{3}$.

One natural generalization where all goes through is to consider a finite group $G$ and a normal subgroup $N$ contained in the center of the group. The factor group $F=G / N$ has elements labeled $1, \sigma, \tau, \ldots$. We may choose coset representatives $t(1)=1, t(\sigma), t(\tau), \ldots$ and any $g=n t(\sigma)$, uniquely. While sometimes $t(\sigma) t(\tau)=$ $t(\sigma \tau)$, in many cases this fails, but we may choose correction factors $f(\sigma, \tau)$ in $N$ (often called a "factor set") so that $t(\sigma) t(\tau)=t(\sigma \tau) f(\sigma, \tau)$. Once the $t(\sigma)$ are chosen, the $f(\sigma, \tau)$ are forced.

Example 17. If $G=C_{100}, N=C_{10}$ (thought of as a subgroup $\{0,10,20, \ldots, 90\}$ ), the natural choice of coset representatives for $G / N \cong\{0,1, \ldots, 9\}$ is $t(i)=i \in G$. Of course,

$$
t(i)+t(j)=t(i+j)+f(i, j), \quad \text { with } f(i, j)= \begin{cases}1 & \text { if } i+j \geq 10, \\ 0 & \text { if } 0 \leq i+j<10 .\end{cases}
$$


It is natural to ask if the choice of cosets matters. To see that it can, consider $C_{10}$ in $C_{100}$ and choose coset representatives as $0,11,22,43,44,45,46,47,48,49$. The sum of 11 and 22 requires a carry of 90 .

A lovely exposition of carries as cocycles is in [45].

If $g=n t(\sigma)$ is chosen uniformly at random, then $n$ and $t(\sigma)$ are independent and uniform. Multiplying a sequence of $g_{i}=n_{i} t_{i}$ can be done by first multiplying the $t_{i}$, keeping track of the carries, and then multiplying the $n_{i}$ and carries in any order. Of course, here the carries are in $N$, not necessarily binary.

Given $t_{1}, t_{2}, \ldots, t_{k}$, we may form a two-column array with $t_{i}$ in the first column, the successive remainders $r_{1}, r_{2}, \ldots, r_{k}$ in the second column and "carries" $f_{1}, f_{2}, \ldots, f_{k-1}$ (elements in $N$ ) placed in between.

Lemma 6.1. If coset representatives $t_{i}, 1 \leq i \leq k$, are chosen uniformly, then

(1) the remainder process $r_{i}, 1 \leq i \leq k$, is uniform and independent, and

(2) the carries $f_{i}, 1 \leq i \leq k-1$, form a stationary, one-dependent, two-block factor.

Proof.

(1) Since $r_{1}=t_{1}, r_{1}$ is uniform. Successive $r_{i}$ are formed by multiplying $r_{i-1}$ by $t_{i}$. There is a unique choice of $t_{i}$ giving $r_{i}$, so $r_{i}$ is uniform and independent of $r_{1}, \ldots, r_{i-1}$.

(2) Consider two successive remainders and the unique $t$ giving rise to them:

$$
\begin{array}{cc} 
& r_{i} \\
t_{i+1} & r_{i+1}
\end{array}
$$

Since $t_{i}$ is uniquely determined, $r_{i} t_{i+1}=r_{i+1} f\left(r_{i}, t_{i+1}\right)$ is uniquely determined. It follows that the $f_{i}$ process is a two-block process: generate $r_{1}, r_{2}, \ldots$ uniformly and independently, set $f_{i}=h\left(r_{i}, r_{i+1}\right)$, with $h\left(r, r^{\prime}\right)=f(r, t)$, where $r^{-1} r^{\prime} \in t N$ determines $t$ uniquely. Because twoblock processes are one-dependent, this completes the proof.

Corollary 6.2. With the notation of the lemma, define a binary process $B_{1}, \ldots$, $B_{k-1}$ as

$$
B_{i}= \begin{cases}1 & \text { if } f_{i} \neq \mathrm{id}, \\ 0 & \text { if } f_{i}=\mathrm{id} .\end{cases}
$$

Then $\left\{B_{i}\right\}$ is a stationary, one-dependent, two-block factor.

Example 18. As in Example 16, let $G=Q_{8}, N=\{ \pm 1\}, F=G / N \cong C_{2} \times C_{2}$. The natural choice of coset representatives $\{1, i, j, k\}$ gives rise to the following twoblock representation: choose $U_{1}, U_{2}, \ldots$ uniformly and independently in $\{1, i, j, k\}$. Let $B_{i}=h\left(U_{i}, U_{i+1}\right)$ with $h(1, x)=1($ all $x), h(x, 1)=-1(x \neq 1), h(i, j)=$ $h(j, k)=h(k, i)=-1, h(i, k)=h(k, j)=h(j, i)=1, h(x, x)=1,(x \neq 1)$.

Example 19 (Dihedral group). Let $D_{8}=\left\langle x, y: x^{2}=y^{2}=(x y)^{4}=1\right\rangle$. If $z=x y$, this 8-element group has center $N=\left\{1, z^{2}=-1\right\}$. Choosing coset representatives 
$1, x, y, z$, the cosets multiply as

\begin{tabular}{c|cccc} 
& 1 & $x$ & $y$ & $z$ \\
\hline 1 & 1 & $x$ & $y$ & $z$ \\
$x$ & $x$ & 1 & $z$ & $y$ \\
$y$ & $y$ & $-z$ & 1 & $-x$ \\
$z$ & $z$ & $-y$ & $x$ & -1
\end{tabular}

From this, elementary manipulations show that $P(i$ ones in a row $)=1 / 4^{i}$. Thus, the carries process is independent with $P\left(B_{i}=1\right)=1 / 4, P\left(B_{i}=0\right)=3 / 4$ for all $i$.

We mention that $D_{8}$ can also be represented as the extension of the normal subgroup $C_{4}$ by the factor group $C_{2}$. Here, coset representatives for $C_{4}$ can be chosen so that there are no carries (i.e., the extension "splits").

Example 20 (Extensions of $C_{2}$ by $C_{m}$ ). A central extension of $N=C_{2}$ by $C_{m}$ is abelian. It follows that when $m$ is odd, $G=C_{2 m}$ is the only central extension of $N=C_{2}$ by $C_{m}$, and when $m$ is even, there are two central extensions $C_{2} \times C_{m}$ and $C_{2 m}$. The extension $C_{2} \times C_{m}$ splits and choosing $\{(0, i), 0 \leq i \leq m-1\}$ as the coset representatives, there are no carries. For $C_{2 m}$, with $C_{2} \cong\{0, m\}$, choose coset representatives $0,1,2, \ldots, m-1$. Thus

$$
f(i, j)= \begin{cases}1 & \text { if } i+j \geq m, \\ 0 & \text { if } 0 \leq i+j<m .\end{cases}
$$

As for the usual addition, there is a carry if and only if there is a descent in the remainder column. Thus

$$
P\left(B_{1}=B_{2}=\cdots=B_{i-1}=1\right)=\frac{\left(\begin{array}{c}
m \\
i
\end{array}\right)}{m^{i}} .
$$

Example 21. Let $G=C_{2} \times C_{2} \times C_{2}$ and let $N=\{(0,0,0),(1,1,1)\}$. With coset representatives $(0,0,0),(1,0,0),(0,1,0),(1,1,0)$, there are never carries, but with coset representatives $(0,0,0),(1,0,0),(0,1,0),(0,0,1)$, there are carries. This example shows that the one-dependent process $B_{1}, B_{2}, \ldots$ depends not only on $G$ and $N$, but also on the choice of coset representatives.

We have not embarked on a systematic study of carries for finite groups and believe that there is much more to do. We do note that by a result in the next section (Theorem [7.1), the above processes, being one-dependent, are determinantal.

The basic carries argument works for infinite groups as well. Let $G$ be a locally compact group and $H$ a closed normal subgroup. Suppose that $H$ is in the center of $G$ and that $G / H$ is compact. Choose coset representatives $t(\sigma) \in G$ for $\sigma \in G / H$. As in the finite case, these define factor sets $f(\sigma, \tau)$ by $t(\sigma) t(\tau)=t(\sigma \tau) f(\sigma, \tau)$. Write $g=n t$. Since $G / H$ is compact, it has an invariant probability measure. Choosing $t_{1}, t_{2}, \ldots$ independently from this measure and multiplying as above gives remainders and a carries process. Just as above, the remainders are independent and uniformly distributed in $G / H$ and the carries process (with values in $N$ ) is a one-dependent, two-block factor process.

Example 22. A lovely instance of this setup explains a classical identity. We begin with the motivation and then translate. Let $\sigma$ be a permutation with number of descents $d(\sigma)$. It is known that, for $\sigma$ chosen from the uniform distribution on $S_{n}$,

$$
P(d(\sigma)=j)=P\left(j \leq U_{1}+\cdots+U_{n}<j+1\right) .
$$


On the right, $U_{1}, U_{2}, \ldots, U_{n}$ are independent uniforms on $[0,1]$. The density and distribution function for $U_{1}+\cdots+U_{n}$ was derived by Laplace; see [31. Foata 33. proved (6.1) by combining Laplace's calculation with an identity of Worpitzky. Richard Stanley [72] gives a bijective proof involving an elegant dissection of the $n$ dimensional hypercube. The following "proof from the book", which is a continuous version of our carries argument from Section 1, appears in a paper of Jim Pitman 60] and also as a hint to Problem 6.65 in [40. Form two columns

$\begin{array}{cr}U_{1} & V_{1} \\ U_{2} & V_{2} \\ \cdot & \cdot \\ \cdot & . \\ U_{n} & V_{n}\end{array}$

On the left are independent uniforms on $[0,1]$. On the right are their remainders when added $\bmod 1$; so $V_{1}=U_{1}, V_{2}=U_{1}+U_{2}(\bmod 1), \ldots$ The $V_{i}$ are similarly independent uniforms on $[0,1]$. Place a dot at position $i$ every time the partial sum $U_{1}+\cdots+U_{i+1}$ crosses an integer. Call these dots carries. As in the discrete case, there is a dot at position $i$ if and only if there is a descent $V_{i+1}<V_{i}$. The number of dots is the integer part of $U_{1}+\cdots+U_{n}$ and also the number of descents, proving (6.1). Of course, the distribution of the descent process is the same as the carries process.

In the language of group theory, let $G=\mathbb{R}, N=\mathbb{Z}$, and $G / N \cong S_{1}$, the circle group. Choose coset representatives as $[0,1)$ and factor sets in $\{0,1\}$.

\section{Proofs and generalizations}

This section proves two of our main results for general one-dependent processes (we do not assume stationarity in this section). In Subsection 7.1, it is shown that all one-dependent point processes on $\mathbb{Z}$ are determinantal, a result which is new even in the stationary case. Subsection 7.2 proves that a point process $P$ on a finite set $\mathcal{X}$, with $P$ given as a certain-shaped determinant, is one-dependent and determinantal. This covers quite a few examples from previous sections and is particularly useful in situations (such as Example 12) where the one-dependence is not a priori obvious.

7.1. One-dependent processes are determinantal. For a random point process on a discrete set $\mathcal{X}$, we define the correlation function $\rho$ by

$$
\rho(A)=P\{S: S \supseteq A\} .
$$

Then one-dependence on a segment of $\mathbb{Z}$ is equivalent to the condition that $\rho(X \cup$ $Y)=\rho(X) \rho(Y)$ whenever $\operatorname{dist}(X, Y) \geq 2$.

Theorem 7.1. Any one-dependent point process on a segment of $\mathbb{Z}$ is determinantal. Its correlation kernel can be written in the form $K(x, y)=$

0

$$
-1
$$$$
\sum_{r=1}^{y-x+1}(-1)^{r-1} \sum_{x=l_{0}<l_{1}<\cdots<l_{r}=y+1} \rho\left(\left[l_{0}, l_{1}\right)\right) \rho\left(\left[l_{1}, l_{2}\right)\right) \cdots \rho\left(\left[l_{r-1}, l_{r}\right)\right) \quad \text { if } x \leq y .
$$

if $x-y \geq 2$,

if $x-y=1$,

Here the notation $[a, b)$ stands for $\{a, a+1, \ldots, b-1\}$. 
For example, $K(x, x)=\rho(\{x\}), K(x, x+1)=\rho(\{x, x+1\})-\rho(\{x\}) \rho(\{x+1\})$, etc.

Proof. By one-dependence, it is enough to verify that with $K$ as above,

$$
\operatorname{det}[K(x+i, x+j)]_{i, j=0}^{y-x}=\rho([x, y+1))
$$

for any $x \leq y$.

We use induction on $y-x$. For $y=x$ the statement is trivial. Otherwise, one has that

$$
\operatorname{det}[K(x+i, x+j)]_{0}^{y-x}=\operatorname{det}\left[\begin{array}{cccc}
K(x, x) & K(x, x+1) & \cdots & K(x, y) \\
-1 & K(x+1, x+1) & \cdots & \vdots \\
& -1 & \ddots & \vdots \\
& & -1 & K(y, y)
\end{array}\right] .
$$

When expanding this determinant, various numbers of -1 's from the subdiagonal can be used. Observe that if we do not use the -1 in position $(i, i-1)$, then we can compute the corresponding contribution, because if we replace that -1 by 0 , the determinant splits into the product of two, each of which is computable by the induction hypothesis.

Similarly, if we insist on not using several -1 's, then the contribution is the product of several determinants. Thus we obtain by inclusion-exclusion that

$$
\begin{aligned}
\operatorname{det}[K(x+i, x+j)]_{0}^{y-x} & =K(x, y)+\sum_{x<l<y+1} \rho([x, l)) \rho([l, y+1)) \\
& -\sum_{x<l_{1}<l_{2}<y+1} \rho\left(\left[x, l_{1}\right)\right) \rho\left(\left[l_{1}, l_{2}\right)\right) \rho\left(\left[l_{2}, y+1\right)\right)+\cdots .
\end{aligned}
$$

The $K(x, y)$ term corresponds to using all -1 's, the sum over $l$ corresponds to not using a -1 at least at location $(l, l-1)$, the sum over $l_{1}, l_{2}$ corresponds to not using $\mathrm{a}-1$ at least at locations $\left(l_{j}, l_{j}-1\right), j=1,2$, etc. Our definition of $K(x, y)$ is such that the right-hand side of (7.1) is equal to $\rho([x, y+1))$, as desired.

To state some corollaries of Theorem [7.1, we use the concept of particle-hole involution. Essentially, given a subset $\mathcal{N}$ of $\mathcal{X}$, the involution maps a point configuration $S \subset \mathcal{X}$ to $S \triangle \mathcal{N}$ (here $\triangle$ is the symbol for symmetric difference). This map leaves intact the particles of $S$ outside of $\mathcal{N}$, and inside $\mathcal{N}$ it loses the particles and picks up the "holes" (points of $\mathcal{N}$ free of particles).

Corollary 7.2. The class of one-dependent processes is closed under the operations of particle-hole involution on any fixed subset, intersections of independent processes, and unions of independent processes.

Proof. For intersections, the claim follows from the definitions.

For particle-hole involutions, note that the property of being one-dependent follows from the kernel being 0 on the second subdiagonal and below. On the other hand, for determinantal point processes the "complementation principle" 6, Sec. A.3] says that the particle-hole involution can be implemented by the following change in the kernel:

$$
\left[\begin{array}{cc}
A & B \\
C & D
\end{array}\right] \rightarrow\left[\begin{array}{cc}
A & B \\
-C & I-D
\end{array}\right]
$$


where the block structure corresponds to the splitting into the noninverted and inverted parts. Clearly, this keeps the property of having 0's on the second subdiagonal and below intact.

Finally, unions can be reduced to intersections by the particle-hole involution.

Remark. The class of determinantal point processes is not closed under intesections/unions.

Let us now look at the translation-invariant case. Then $\rho([x, y))=\rho_{y-x}$ is a function of $y-x$ only, and $\rho_{k}$ is the chance of $k$ consecutive ones. Set

$$
R(z)=1+z+\sum_{k \geq 1} \rho_{k} z^{k+1}
$$

Corollary 7.3. In the translation-invariant case, the kernel $K(x, y)=k(y-x)$ is also translation invariant, and

$$
\sum_{n \in \mathbb{Z}} k(n) z^{n}=\frac{1}{1-R(z)}
$$

Proof.

$$
\begin{aligned}
\frac{1}{1-R(z)} & =-\frac{1}{z} \cdot \frac{1}{1+\rho_{1} z+\rho_{2} z^{2}+\cdots} \\
& =-\frac{1}{z}\left[1-\sum_{m \geq 1} \rho_{m} z^{m}+\left(\sum_{m \geq 1} \rho_{m} z_{m}\right)^{2}-\cdots\right] \\
& =-\frac{1}{z}+\frac{1}{z}\left[\sum_{m \geq 1} \rho_{m} z^{m}-\left(\sum_{m \geq 1} \rho_{m} z^{m}\right)^{2}+\cdots\right]
\end{aligned}
$$

which agrees with the formula of Theorem 7.1 .

The generating function $R(z)$ also behaves well with respect to the particle-hole involution on $\mathbb{Z}$.

Proposition 7.4. The particle-hole involution on $\mathbb{Z}$ with $\mathcal{N}=\mathbb{Z}$ replaces $R(z)$ by $1 / R(-z)$.

Proof. We have

$$
\frac{1}{1-1 / R(-z)}=\frac{R(-z)}{R(-z)-1}=1-\frac{1}{1-R(-z)} .
$$

Hence by Corollary [7.3, changing $R(z)$ to $\tilde{R}(z)=1 / R(-z)$ leads to the following change in the correlation kernel:

$$
\tilde{k}(n)=\delta_{0, n}-(-1)^{n} k(n) .
$$

This is equivalent to $\tilde{K}(x, y)=\delta_{x, y}-\frac{(-1)^{x}}{(-1)^{y}} K(x, y)$. This is the same as $K \rightarrow I-K$, which corresponds to the particle-hole involution [6, Sec. A.3].

Example 23. The descent process on $\mathbb{Z}$ (Example 11) corresponds to $\rho_{m}=\frac{1}{(m+1) !}$; thus $R(z)=e^{z}$. The particle-hole involution is given by $\tilde{R}(z)=1 / e^{-z}=e^{z}$, which is the same. 
Example 24. The intersection of $r$ independent descent (or ascent) processes on $\mathbb{Z}$ corresponds to $\rho_{m}=\frac{1}{(m+1) ! r} ;$ hence

$$
R_{\cap}^{(r)}(z)=\sum_{m \geq 0} \frac{z^{m}}{(m !)^{r}} .
$$

The correlation kernel is given by $\sum_{n \in \mathbb{Z}} k(n) z^{n}=\frac{1}{1-R_{\Pi}^{(r)}(z)}$.

Example 25. The union of $r$ independent descent processes is the particle-hole involution of Example 24. Thus $R_{\cup}^{(r)}(z)=1 / R_{\cap}^{(r)}(-z)$ and

$$
\sum_{n \in \mathbb{Z}} k(n) z^{n}=\frac{1}{1-1 / R_{\cap}^{(r)}(-z)} .
$$

Replacing $z$ by $-z$ doesn't affect correlations, and we recover Example 15

7.2. A class of determinantal processes. For any $n=2,3, \ldots$, consider a probability measure $P_{n}$ on all subsets $S=\left\{s_{1}<s_{2}<\cdots<s_{k}\right\} \subseteq[n-1]$ given by

$$
P_{n}(S)=h(n) \operatorname{det}\left[e\left(s_{i}, s_{j+1}\right)\right]_{i, j=0}^{k},
$$

for some $h: \mathbb{N} \rightarrow \mathbb{C}$ and $e: \mathbb{N} \times \mathbb{N} \rightarrow \mathbb{C}$ with the notation $s_{0}=0, s_{k+1}=n$. We assume that $e(i, j)=0$ for $i>j$, and that $e(i, i)=1, e(i, i+1)>0$ for all $i$.

Theorem 7.5. If (7.2) holds for some fixed $n$ with $e(i, j)=0$ for $i>j$ and $e(i, i)=1, e(i, i+1)>0$ for all $i$, then $P_{n}$ is a determinantal, one-dependent process with correlation functions

$$
\rho(A)=P_{n}\{S: S \supseteq A\}=\operatorname{det}\left[K\left(a_{i}, a_{j}\right)\right]_{i, j=1}^{m} \quad \text { for } A=\left\{a_{1}, a_{2}, \ldots, a_{m}\right\},
$$

with correlation kernel

$$
K(x, y)=\delta_{x, y}+\left(E^{-1}\right)_{x, y+1},
$$

where $E$ is the upper triangular matrix $E=[e(i-1, j)]_{i, j=1}^{n}$,

$$
E=\left[\begin{array}{cccc}
e(0,1) & e(0,2) & \cdots & e(0, n) \\
& e(1,2) & \cdots & e(1, n) \\
& & \ddots & \vdots \\
& & & e(n-1, n)
\end{array}\right] .
$$

In addition, $h(n)=(\operatorname{det} E)^{-1}=(e(0,1) e(1,2) \cdots e(n-1, n))^{-1}$.

Corollary 7.6. Assume further that $e(i, j)=e(j-i)$. Then the point process is stationary. If $\hat{e}(z)=\sum_{l=0}^{\infty} e(l) z^{l}$, then $K(x, y)=k(y-x)$ and

$$
\hat{k}(z):=\sum_{l=-\infty}^{\infty} k(l) z^{l}=\frac{1}{1-1 / \hat{e}(z)} .
$$

Proof of Theorem 7.5. Set $L=\left[e(i-1, j)+\delta_{i-1, j}\right]_{i, j=1}^{n}$. Thus $L$ appears as

$$
L=\left[\begin{array}{cccc}
e(0,1) & e(0,2) & \cdots & e(0, n) \\
1 & e(1,2) & \cdots & \vdots \\
& 1 & \ddots & \vdots \\
& & 1 & e(n-1, n)
\end{array}\right]
$$


For any function $f:\{1, \ldots, n-1\} \rightarrow \mathbb{C}$, by (7.2),

$$
\begin{aligned}
& \mathbb{E}\left(\prod_{s_{i} \in S} f\left(s_{i}\right)\right) \\
& =\sum_{\substack{0<s_{1}<\cdots<s_{k}<n \\
k=0, \cdots, n-1}} P_{n}\left(\left\{s_{1}<s_{2}<\cdots<s_{k}\right\}\right) f\left(s_{1}\right) \cdots f\left(s_{k}\right) \\
& =h(n) \sum_{\substack{0<s_{1}<\cdots<s_{k}<n \\
k=0, \ldots, n-1}} \operatorname{det}\left[L\left(\begin{array}{c}
1, s_{1}+1, \ldots, s_{k}+1 \\
s_{1}, \ldots, s_{k}, n
\end{array}\right)\right] f\left(s_{1}\right) \cdots f\left(s_{k}\right) \\
& =h(n) \cdot \operatorname{det}\left[\begin{array}{ccccc}
f(1) e(0,1) & f(2) e(0,2) & \cdots & f(n-1) e(0, n-1) & e(0, n) \\
f(1)-1 & f(2) e(1,2) & \cdots & f(n-1) e(1, n-1) & e(1, n) \\
& f(2)-1 & \ddots & f(n-1) e(2, n-1) & e(2, n) \\
& & \ddots & \cdots & \cdots \\
& & & f(n-1)-1 & e(n-1, n)
\end{array}\right]
\end{aligned}
$$

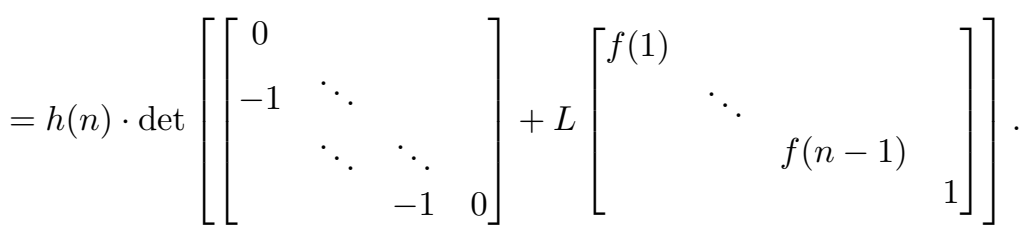

This is the generating functional of $P_{n}$. In the second equality, the determinant is of the minor of $L$ with rows $1, s_{1}+1, \ldots, s_{k}+1$ and columns $s_{1}, \ldots, s_{k}, n$. In the third inequality, the $2^{n-1}$ possible summands correspond to choosing which of the first $n-1$ matrix columns use the -1 coming from $f(i)-1$ in the determinant expansion.

If $f=1+g$, the generating functional can be expressed in terms of the correlation functions:

$$
\begin{aligned}
& 1+\sum_{\substack{s_{1}<\cdots<s_{m} \\
m=1,2, \ldots}} \rho_{m}\left(s_{1}, \ldots, s_{m}\right) g\left(s_{1}\right) \cdots g\left(s_{m}\right) \\
= & \mathbb{E}\left[\prod_{s_{i} \in S}\left(1+g\left(s_{i}\right)\right)\right] \\
= & h(n) \operatorname{det}\left(E+L\left[\begin{array}{llll}
g(1) & & & \\
& \ddots & & \\
& & g(n-1) & \\
& & 0
\end{array}\right]\right) \\
= & h(n) \operatorname{det}(E) \cdot \operatorname{det}\left(I+E^{-1} L \cdot\left[\begin{array}{llll}
g(1) & & \\
& \ddots & & \\
& & g(n-1) & \\
& & & 0
\end{array}\right]\right) .
\end{aligned}
$$

This holds for all $g$. First take $g=0$ to see that $h(n) \operatorname{det}(E)=1$. Next note the expansion: if $M$ is $n \times n$, then $\operatorname{det}[I+M]=\sum_{S} \operatorname{det}(M(S))$ with the sum over all $2^{n}$ subsets of $[n]$, and $M(S)$ the minor with rows and columns in $S$. This gives that 


$$
\begin{aligned}
& \rho_{m}\left(s_{1}, \ldots, s_{m}\right)=\operatorname{det}\left[K\left(s_{i}, s_{j}\right)\right]_{i, j=1}^{m} \text {, where } K(x, y)=\left(E^{-1} L\right)_{x y} \text {. Then } \\
& E^{-1} L=E^{-1}\left(\left[\begin{array}{cccc}
0 & & & \\
1 & \ddots & & \\
& \ddots & \ddots & \\
& & 1 & 0
\end{array}\right]+E\right)=I+E^{-1}\left[\begin{array}{cccc}
0 & & & \\
1 & \ddots & & \\
& \ddots & \ddots & \\
& & 1 & 0
\end{array}\right],
\end{aligned}
$$

and the proof of (7.3) is complete.

Finally, we note that since $K(x, y)$ vanishes below the first subdiagonal, $P_{n}$ is one-dependent.

Proof of corollary. Here $E$ is a Toeplitz matrix with symbol $(\hat{e}(z)-1) / z$. It is triangular, so $E^{-1}$ is a Toeplitz matrix with symbol $z /(\hat{e}(z)-1)$. Thus $K$ is a Toeplitz matrix with symbol $\hat{k}(z)=1+\frac{1}{\hat{e}(z)-1}=\frac{1}{1-(\hat{e}(z))^{-1}}$.

We also note that in the translation-invariant case, there is an expression for $P_{n}(S)$ in terms of skew Schur functions of ribbon type.

Corollary 7.7. As in Corollary 17.6, assume further that $e(i, j)=e(j-i)$. Let $\lambda$ and $\mu$ be the partitions defined by

$$
\lambda_{i}=n-s_{i-1}-k+i-1, \mu_{i}=n-s_{i}-k+i-1, \quad 1 \leq i \leq k+1 .
$$

Let $\lambda^{\prime}, \mu^{\prime}$ denote the transposed partitions of $\lambda$ and $\mu$ and let $s_{\lambda^{\prime} / \mu^{\prime}}$ denote the corresponding skew-Schur function, obtained by specializing the elementary symmetric functions $e_{i}$ to equal $e(i)$. Then $P_{n}(S)=\frac{1}{e(1)^{n}} s_{\lambda^{\prime}} / \tau^{\prime}$.

Proof. By assumption, formula (7.2) becomes

$$
P_{n}(S)=h(n) \cdot \operatorname{det}\left(e_{s_{j+1}-s_{i}}\right)_{i, j=0}^{k} .
$$

Now use the argument of Theorem 4.2 , together with the identification of the normalizing constant $h(n)=1 / e(1)^{n}$ in Theorem 7.5.

Remarks.

(1) Another approach to Theorem 7.5 is via the theory of conditional $L$-ensembles in Borodin-Rains [7, Prop. 1.2].

(2) In the translation-invariant case $e(i, j)=e(j-i)$, Stanley [70, p. 90, Ex. 14] shows that

$$
P_{n}([n-1])=\text { chance that all sites are occupied }
$$

is the coefficient of $z^{n}$ in the power series $h(n) \hat{e}(-z)^{-1}$. To prove this using symmetric function theory, note from Corollary 7.7 that

$$
P_{n}([n-1])=h(n) \cdot s_{(n)} .
$$

Here the Schur function $s_{(n)}=h_{n}$ (where $h_{n}$ is the $n$th complete homogeneous symmetric function and $h(n)$ is the normalizing constant in (7.2)). If $E(z)=\sum_{r=0}^{\infty} e_{r} z^{r}$ is the generating function for elementary symmetric functions and $H(z)=\sum_{r=0}^{\infty} h_{r} z^{r}$, Macdonald [54, p. 21, (2.6)] shows that $H(z) E(-z)=1$. This gives Stanley's formula since in Corollary 7.7 the value of $e_{r}$ is $e(r)$. 
Example 26 (Descents in a random sequence). From Fact 5 of Section 2, we are in the Toeplitz case with $e(j)=\left(\begin{array}{c}j+b-1 \\ b-1\end{array}\right), \hat{e}(z)=(1-z)^{-b}$. Applying Corollary 7.6 yields Theorem 3.1

Example 27 (Descents in a uniform permutation). From MacMahon's formula (5.1), we are again in the Toeplitz case with $e(j)=1 / j !, \hat{e}(z)=e^{z}$. As in Example3, one can replace $z$ by $-z$ without changing determinants or the correlation functions. This proves Theorem 5.1 .

Example 28 (Descents in a nonuniform permutation). From Stanley's formula (part (a) of Proposition 5.2), we are again in the Toeplitz case with $e(j)=1 / j !_{q}$ ( $q$-factorial). An identity of Euler allows one to write

$$
\hat{e}(z)=\prod_{m \geq 0} \frac{1}{1-z(1-q) q^{m}}
$$

when $0<q<1,|z|<1$, but the elementary description of the correlation functions given in Proposition 5.2 seems more useful.

\section{ACKNOWLEDGMENTS}

Borodin was partially supported by NSF grant DMS 0707163. Diaconis was partially supported by NSF grant DMS 0505673. Fulman was partially supported by NSF grant DMS 0802082 and NSA grant H98230-08-1-0133. We thank David Eisenbud, Don Knuth, and Peter Winkler for useful comments.

\section{ABOUt THE AUTHORS}

Alexei Borodin is the Gordon M. Binder/Amgen Professor of Mathematics at the California Institute of Technology. Persi Diaconis is the Mary B. Sunseri Professor of Mathematics and Statistics at Stanford University. Jason Fulman is professor of mathematics at the University of Southern California.

\section{REFERENCES}

1. Ando, T., Totally positive matrices, Linear Algebra Appl. 90 (1987), 165-219. MR884118 (88b:15023)

2. Arnold, B. C., Balakrishnan, N. and Nagaraja, H. N., A first course in order statistics, Wiley Series in Probability and Mathematical Statistics: Probability and Mathematical Statistics. John Wiley \& Sons, New York, 1992. MR1178934 (94a:62076)

3. Billera, L. J., Thomas, H. and van Willigenburg, S., Decomposable compositions, symmetric quasisymmetric functions and equality of ribbon Schur functions, Adv. Math. 204 (2006), 204-240. MR2233132 (2007b:05207)

4. Borodin, A., Loop-free Markov chains as determinantal point processes, Ann. Inst. Henri Poincaré Probab. Stat. 44 (2008), no. 1, 19-28. MR2451569 (2010a:60239)

5. Borodin, A., Determinantal point processes, arXiv:0911.1153 (2009), to appear in Oxford Handbook of Random Matrix Theory.

6. Borodin, A., Okounkov, A. and Olshanski, G., Asymptotics of Plancherel measures for symmetric groups, J. Amer. Math. Soc. 13 (2000), 481-515 (electronic). MR 1758751 (2001g:05103)

7. Borodin, A. and Rains, E. M., Eynard-Mehta theorem, Schur process, and their Pfaffian analogs, J. Stat. Phys. 121 (2005), 291-317. MR2185331 (2006k:82039)

8. Bradley, R. C., On a stationary, triple-wise independent, absolutely regular counterexample to the central limit theorem, Rocky Mountain J. Math. 37 (2007), 25-44. MR 2316436 (2008a:60050) 
9. Brenti, F., Unimodal, log-concave and Pólya frequency sequences in combinatorics, Mem. Amer. Math. Soc. 81 (1989), no. 413, viii+106. MR.963833 (90d:05014)

10. Broman, E. I., One-dependent trigonometric determinantal processes are two-block-factors, Ann. Probab. 33 (2005), 601-609. MR2123203 (2005k:60116)

11. Burton, R. and Pemantle, R., Local characteristics, entropy and limit theorems for spanning trees and domino tilings via transfer-impedances, Ann. Probab. 21 (1993), 1329-1371. MR.1235419 (94m:60019)

12. Chebikin, D., Polytopes, generating functions, and new statistics related to descents and inversions in permutations, Ph.D. thesis, Department of Mathematics, MIT, 2008.

13. Chen, L. H. Y. and Shao, Q., Normal approximation under local dependence, Ann. Probab. 32 (2004), 1985-2028. MR2073183 (2005f:60054)

14. Cifarelli, D. M. and Fortini, S., A short note on one-dependent trigonometric determinantal probability measures. Technical report, Istituto di Metodi Quantitativi, Università Bocconi, 2005.

15. Comtet, L., Advanced combinatorics, D. Reidel, Dordrecht, 1974. MR0460128 (57:124)

16. Critchlow, D. E., Metric methods for analyzing partially ranked data, Lecture Notes in Statistics, 34. Springer-Verlag, Berlin, 1985. MR818986 (87c:62044)

17. Cutler, A. and McShane, R., The Trachtenberg speed system of basic mathematics, Doubleday \& Co., Garden City, N.Y., 1960. MR.0117137 (22:7920)

18. Daley, D. J. and Vere-Jones, D., An introduction to the theory of point processes, Springer Series in Statistics, Springer-Verlag, New York, 1988. MR950166 (90e:60060)

19. Dembo, A. and Rinott, Y., Some examples of normal approximations by Stein's method, in: Random discrete structures (Minneapolis, MN, 1993), pp. 25-44, IMA Vol. Math. Appl. 76, Springer, New York, 1996. MR1395606 (97f:60051)

20. Diaconis, P., Group representations in probability and statistics, Institute of Mathematical Statistics Lecture Notes-Monograph Series, 11. Institute of Mathematical Statistics, Hayward, CA, 1988. MR964069 (90a:60001)

21. Diaconis, P. and Fulman, J., Carries, shuffling, and symmetric functions, Adv. Appl. Math 43 (2009), 176-196. MR 2531920

22. Diaconis, P. and Hanlon, P., Eigen-analysis for some examples of the Metropolis algorithm, in: Hypergeometric functions on domains of positivity, Jack polynomials, and applications, Contemp. Math. 138, Amer. Math. Soc., Providence, RI, 1992, 99-117. MR1199122 (94b:05205)

23. Diaconis, P., McGrath, M., and Pitman, J., Riffle shuffles, cycles and descents, Combinatorica 15 (1995), 11-29. MR.1325269 (96g:05009)

24. Diaconis, P. and Ram, A., Analysis of systematic scan Metropolis algorithms using IwahoriHecke algebra techniques, Michigan Math. J. 48 (2000), 157-190. MR1786485 (2001j:60132)

25. Doubilet, P., Rota, G.-C., and Stanley, R., On the foundations of combinatorial theory. VI. The idea of generating function, in: Proceedings of the Sixth Berkeley Symposium on Mathematical Statistics and Probability Vol. II: Probability theory, pp. 267-318. Univ. California Press, Berkeley, Calif., 1972. MR0403987 (53:7796)

26. Dyson, F. J., Statistical theory of the energy levels of complex systems. I, J. Mathematical Phys. 3 (1962), 140-156. MR0143556 (26:1111)

27. Dyson, F. J., Statistical theory of the energy levels of complex systems. II, J. Mathematical Phys. 3 (1962), 157-165. MR0143557 (26:1112)

28. Dyson, F. J., Statistical theory of the energy levels of complex systems. III, J. Mathematical Phys. 3 (1962), 166-175. MR0143558 (26:1113)

29. Edrei, A., Proof of a conjecture of Schoenberg on the generating function of a totally positive sequence, Canadian J. Math. 5 (1953), 86-94. MR0053176 (14:732f)

30. Evans, D. L., Leemis, L. M. and Drew, J. H., The distribution of order statistics for discrete random variables with applications to bootstrapping, INFORMS J. Comput. 18 (2006), 19-30. MR 2205744

31. Feller, W., An introduction to probability theory and its applications. Vol. II., 2nd ed., John Wiley and Sons, New York-London-Sydney, 1971. MR0270403 (42:5292)

32. Fligner, M. A. and Verducci, J. S. (eds.), Probability models and statistical analyses for ranking data, Lecture Notes in Statistics 80. Springer-Verlag, New York, 1993. MR.1237197 (94c:62007) 
33. Foata, D., Distributions Eulériennes et Mahoniennes sur le groupe des permutations, in: Higher Combinatorics, M. Aigner, editor, page 47. Reidel, Dordrecht/Boston, 1977. MR.519777 (80e:05011)

34. Foulkes, H. O., Enumeration of permutations with prescribed up-down and inversion sequences, Discrete Math. 15 (1976), 235-252. MR0406809 (53:10595)

35. Fröberg, R., Koszul algebras, in: Advances in commutative ring theory (Fez, 1997), 337350, Lecture Notes in Pure and Appl. Math., 205, Dekker, New York, 1999. MR 1767430 (2001i:16046)

36. Fulman, J., Applications of symmetric functions to cycle and increasing subsequence structure after shuffles, J. Algebr. Comb. 16 (2002), 165-194. MR1943587(2004b:05200)

37. Garsia, A. M. and Reutenauer, C., A decomposition of Solomon's descent algebra, Adv. Math. 77 (1989), 189-262. MR.1020585 (91c:20007)

38. Gessel, I. and Reutenauer, C., Counting permutations with given cycle structure and descent set, J. Combin. Theory Ser. A 64 (1993), 189-215. MR.1245159 (95g:05006)

39. Gessel, I. and Viennot, G., Binomial determinants, paths, and hook length formulae, Adv. in Math. 58 (1985), 300-321. MR815360(87e:05008)

40. Graham, R., Knuth, D., and Patashnik, O., Concrete mathematics. A foundation for computer science. Second edition. Addison-Wesley, Reading, MA, 1994. MR1397498 (97d:68003)

41. Harper, L. H., Stirling behavior is asymptotically normal, Ann. Math. Statist. 38 (1967), 410-414. MR0211432 (35:2312)

42. Hoeffding, W. and Robbins, H., The central limit theorem for dependent random variables, Duke Math. J. 15 (1948), 773-780. MR0026771 (10:200e)

43. Hough, J. B., Krishnapur, M., Peres, Y., and Virág, B., Determinantal processes and independence, Probab. Surv. 3 (2006), 206-229 (electronic). MR2216966 (2006m:60068)

44. Hough, J. B., Krishnapur, M., Peres, Y., and Virág, B., Zeros of Gaussian analytic functions and determinantal point processes. University Lecture Series, 51. American Mathematical Society, Providence, RI, 2009. MR 2552864

45. Isaksen, D., A cohomological viewpoint on elementary school arithmetic, Amer. Math. Monthly 109 (2002), 796-805. MR 1933702

46. Janson, S., Some pairwise independent sequences for which the central limit theorem fails, Stochastics 23 (1988), 439-448. MR 943814 (89e:60048)

47. Johansson, K., Random matrices and determinantal processes, Mathematical Statistical Physics, 1-55, Elsevier B. V., Amsterdam 2006.

48. Johansson, K. and Nordenstam, E., Eigenvalues of GUE minors, Electron. J. Probab. 11 (2006), 1342-1371 (electronic). MR2268547 (2008d:60066a)

49. Kenyon, R., Lectures on dimers, in: Statistical mechanics, IAS/Park City Math. Ser., 16, Amer. Math. Soc., Providence, RI, 2009, 191-230. MR2523460

50. Lascoux, A. and Pragacz, P., Ribbon Schur functions, European J. Combin. 9 (1988), 561-574. MR 970392 (89k:05013)

51. Lyons, R., Determinantal probability measures, Publ. Math. Inst. Hautes Études Sci. 98 (2003), 167-212. MR2031202 (2005b:60024)

52. Lyons, R. and Steif, J., Stationary determinantal processes: phase multiplicity, Bernoullicity, entropy, and domination, Duke Math. J. 120 (2003), 515-575. MR2030095 (2004k:60100)

53. Macchi, O., The coincidence approach to stochastic point processes, Advances in Appl. Probability 7 (1975), 83-122. MR0380979 (52:1876)

54. Macdonald, I. G., Symmetric functions and Hall polynomials, Second edition. The Clarendon Press, Oxford University Press, New York, 1995. MR1354144 (96h:05207)

55. MacMahon, P. A., Combinatory analysis. Vols. I, II (bound in one volume). Reprint of An introduction to combinatory analysis (1920) and Combinatory analysis. Vols. I, II (1915, 1916). Dover Publications, Mineola, NY, 2004. MR2417935 (2009e:05002)

56. Mallows, C. L., Non-null ranking models. I, Biometrika 44 (1957), 114-130. MR0087267 $(19: 330 \mathrm{a})$

57. Marden, J. I., Analyzing and modeling rank data, Monographs on Statistics and Applied Probability 64. Chapman \& Hall, London, 1995. MR1346107 (96j:62127)

58. Mehta, M. L., Random matrices, Third edition. Pure and Applied Mathematics (Amsterdam), 142. Elsevier/Academic Press, Amsterdam, 2004. MR2129906 (2006b:82001)

59. Peres, Y. and Virág, B., Zeros of the i.i.d. Gaussian power series: A conformally invariant determinantal process, Acta Math. 194 (2005), 1-35. MR.2231337(2007m:60150) 
60. Pitman, J. W., Probabilistic bounds on the coefficients of polynomials with only real zeros, J. Combin. Theory Ser. A $\mathbf{7 7}$ (1997), 279-303. MR1429082 (97m:05013)

61. Polishchuk, A. and Positselski, L., Quadratic algebras, University Lecture Series, 37. American Mathematical Society, Providence, RI, 2005. MR2177131 (2006f:16043)

62. Reiner, V., Signed permutation statistics, European J. Combin. 14 (1993), 553-567. MR 1248063 (95e:05008)

63. Reiner, V., Shaw, K. M., and van Willigenburg, S., Coincidences among skew Schur functions, Adv. Math. 216 (2007), 118-152. MR2353252(2008m:05303)

64. Reutenauer, C., Free Lie algebras, London Mathematical Society Monographs. New Series, 7. Oxford Science Publications. The Clarendon Press, Oxford University Press, New York, 1993. MR.1231799 (94j:17002)

65. Shirai, T. and Takahashi, Y., Random point fields associated with certain Fredholm determinants, I. Fermion, Poisson and boson point processes, J. Funct. Anal. 205 (2003), 414-463. MR2018415 (2004m:60104)

66. Shirai, T. and Takahashi, Y., Random point fields associated with certain Fredholm determinants, II. Fermion shifts and their ergodic and Gibbs properties, Ann. Probab. 31 (2003), 1533-1564. MR.1989442 (2004k:60146)

67. Solomon, L., A Mackey formula in the group ring of a Coxeter group, J. Algebra 41 (1976), 255-264. MR0444756 (56:3104)

68. Soshnikov, A., Determinantal random point fields, Russian Math. Surveys 55 (2000), 923-975. MR:1799012 (2002f:60097)

69. Stanley, R. P., Binomial posets, Möbius inversion, and permutation enumeration, J. Combinatorial Theory Ser. A 20 (1976), 336-356. MR0409206 (53:12968)

70. Stanley, R. P., Enumerative combinatorics. Vol. 1. Corrected reprint of the 1986 original. Cambridge Studies in Advanced Mathematics, 49. Cambridge University Press, Cambridge, 1997. MR1442260 (98a:05001)

71. Stanley, R. P., Enumerative combinatorics. Vol. 2, Cambridge Studies in Advanced Mathematics, 62. Cambridge University Press, Cambridge, 1999. MR1676282 (2000k:05026)

72. Stanley, R. P., Eulerian partitions of a unit hypercube, in: Higher Combinatorics, M. Aigner, editor, page 47. Reidel, Dordrecht/Boston, 1977.

73. Stanley, R. P., Generalized riffle shuffles and quasisymmetric functions, Ann. Comb. 5 (2001), 479-491. MR1897637 (2003c:60016)

74. Stanley, R. P., The descent set and connectivity set of a permutation, J. Integer Seq. 8 (2005), Article 05.3.8, 9 pp. (electronic). MR2167418 (2006c:05009)

75. Stembridge, J. R., Counterexamples to the poset conjectures of Neggers, Stanley, and Stembridge, Trans. Amer. Math. Soc. 359 (2007), 1115-1128 (electronic). MR 2262844 (2007i:06007)

76. de Valk, V., One-dependent processes: Two-block factors and non-two-block factors, CWI Tract, 85. Stichting Mathematisch Centrum, Centrum voor Wiskunde en Informatica, Amsterdam, 1994. MR1269540 (95h:60056)

77. Wilf, H. S., Algorithms and complexity, second edition, A K Peters, Natick, MA, 2002. MR.1942991 (2003i:68055)

Department of Mathematics, Caltech, Pasadena, California, 91125

Departments of Mathematics and Statistics, Stanford, Palo Alto, California, 94305

Department of Mathematics, University of Southern California, Los Angeles, CalIFORNIA, 90089 